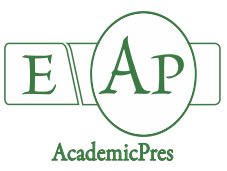

Fan S et al. (2021)

Notulae Botanicae Horti Agrobotanici Cluj-Napoca

Volume 49, Issue 3, Article number 12489

DOI: $10.15835 /$ nbha49312489

Research Article

\title{
Genome-wide investigation of Hydroxycinnamoyl CoA: Shikimate Hydroxycinnamoyl Transferase (HCT) gene family in Carthamus tinctorius $L$.
}

\author{
Sun FAN ${ }^{1 a}$, Naveed AHMAD ${ }^{1,3 b}$, Jin LIBO ${ }^{2,4 c}$, Zhang XINYUE ${ }^{1}$, \\ Ma XINTONG ${ }^{1}$, Nguyen Q.V. HOANG ${ }^{1}$, Ali I. MALLANO ${ }^{3}$, \\ Wang NAN $^{1}$, Yang ZHUODA ${ }^{1}$, Liu XIUMING ${ }^{1 *}$, Yao NA ${ }^{1 *}$
}

\begin{abstract}
${ }^{1}$ Jilin Agricultural University, Ministry of Education Engineering Research Center of Bioreactor and Pharmaceutical Development, Changchun130118,China; porker0927@outlook.com;naveedjlau@gmail.com;2778935775@qq.com; mxt7142020@163.com; viethoangilau@gmail.com;2124136734@qq.com; Xiuming1211@163.com (*corresponding author); nayao1103@jlau.edu.cn (*corresponding author)

${ }^{2}$ Wenzhou University, Institute of Life Sciences, Wenzhou 325035, China;

${ }^{3}$ Shandong Academy of Agricultural Sciences, Biotechnology Research Center, Shandong Provincial Key, Laboratory of Crop Genetic Improvement, Ecology and Physiology, Jinan 250100,China; iamallano@sau.edu.pk;

${ }^{4}$ Wenzhou University, Biomedical Collaborative Innovation Center of Zhejiang Province, Wenzhou, Zhejiang 325035, China a,b,c These authors contributed equally to this work.
\end{abstract}

\begin{abstract}
Hydroxycinnamoyl-CoA: shikimate hydroxycinnamoyl transferase (HCT) is mainly associated with monolignol biosynthesis, a central precursor to producing guaiacyl and syringyl lignins in plants. However, the explicit regulatory mechanism of HCT-mediated monolignol biosynthesis in plants still remained unclear. Here, the genome-wide analysis of the HCT gene family in Carthamus tinctorius as a target for understanding growth, development, and stress-responsive mechanisms was investigated. A total of $82 C t H C T$ genes were identified and characterized. Most of the CtHCTs proteins demonstrated the presence of two common conserved domains, including HXXXD and DFGWG. In addition, the conserved structure of protein motifs, PPI network, cis-regulatory units, and gene structure analysis demonstrated several genetic determinants reflecting the wide range of functional diversity of $\mathrm{CtHCT}$-encoding genes. The observed expression analysis of $C t H C T$ genes in different flowering stages under normal conditions partially highlighted their putative roles in plant growth and development pathways. Moreover, $C t H C T$ genes appeared to be associated with abiotic stress responses as validated by the expression profiling in various flowering phases under light irradiation and MeJA treatment. Altogether, these findings provide new insights into identifying crucial molecular targets associated with plant growth and development and present practical information for understanding abiotic stress-responsive mechanisms in plants.
\end{abstract}

Keywords: abiotic stress; Carthamus tinctorius, expression diversity; HCT gene family; monolignol biosynthesis

Received: 31 August 2021. Received in revised form: 21 Sep 2021. Accepted: 22 Sep 2021. Published online: 27 Sep 2021.

From Volume 49, Issue 1, 2021, Notulae Botanicae Horti Agrobotanici Cluj-Napoca journal uses article numbers in place of the traditional method of continuous pagination through the volume. The journal will continue to appear quarterly, as before, with four annual numbers. 


\section{Introduction}

Carthamus tinctorius is commonly known as safflower or 'bastard saffron', which belongs to the Asteraceae family of the plant kingdom. The increasing demand for its oilseed, which is extremely rich in conjugated linoleic acid, has attracted the attention of plant biologists worldwide. Safflower's oilseed consists of $80 \%$ of octadecadienoic acid, which helps regulate the rate of cholesterol and avert diseases related to cardiovascular channels (Roh et al., 2004). C. tinctorius is radically recommended for its medicinal and economic value in the mainland of China and west Asia. Following multiple phytochemical and pharmacologic investigations on safflower petals, it was discovered that the vital component that provides abundant resources of pharmacogenetic importance is flavonoid. Almost over 5000 types of phenolic compounds and lignin derivatives exist across the plant kingdom in which safflower shares a remarkable reservoir of flavonoids. The widely distributed classes of flavonoids in C. tinctorius mainly include carthamin chalcone glycoside, kaempferol glucosides, hydroxylsafflor yellow A\&B, and quercetin glucosides (Ye and Gao, 2008; Zhang et al., 2011).

Hydroxycinnamoyl CoA: Shikimate Hydroxycinnamoyl Transferase (HCT) gene family is widely known as acyl-CoA-dependent transferases, including various enzymes that utilize the commonly used donner molecule hydroxycinnamoyl-CoAs which catalyse a group of reactions and substrates (Chiang et al., 2018). HCT synthesizes p-coumaroyl shikimate by transferring the p-coumaroyl group from the acyl donor pcoumaroyl-CoA to the acyl acceptor shikimate. It is an essential enzyme in the phenylpropanoid metabolism, conserved across all land plants (Chao et al., 2021; Weng and Chapple, 2010). The downstream pathway catalyses the conversion of phenylalanine into a variety of hydroxycinnamic acids, which are the key precursor molecules of flavonoids, hydroxycinnamic acid conjugates, and lignins (Wang et al., 2015). The metabolic pathways towards lignin and chlorogenic acid (CGA) presumably share common intermediates and enzymes. Various studies in the dicots Solanum lycopersicum (tomato), Solanum tuberosum (potato), Nicotiana tabacum (tobacco), and Cynara cardunculus (globe artichoke) have suggested different routes for CGA biosynthesis in plants (Escamilla-Treviño et al., 2014; Payyavula et al., 2015; Sonnante et al., 2010). The enzyme (HCT) catalyzes CGA formation from caffeoyl CoA and quinic acid in the first pathway. To produce the caffeoyl CoA substrate, 4-coumaroyl CoA is converted to 4-coumaroyl shikimate by (HCT), this shikimate ester receptor molecule is hydroxylated by the enzyme 4-coumaroyl shikimate 3'-hydroxylase $\left(\mathrm{C}^{\prime} \mathrm{H}\right)$, and the caffeoyl shikimate generated is converted to caffeoyl CoA by an HCT enzyme acting in the reverse direction. The second suggested route proceeds by synthesizing 4-coumaroyl quinate by HCT or HQT enzymes, followed by hydroxylation of the coumaroyl moiety by C3' $\mathrm{H}$ (Eudes et al., 2016; Tsai et al., 2006; Wagner et al., 2007).

In vascular plants, the phenylpropanoid pathway is required to synthesize many metabolites, including lignin, which provides mechanical strength to vascular tissues and defense against various stresses (Vanholme et al., 2019; Wang et al., 2015). For instance, low temperature, high salinity, drought, mechanical injury, abscisic acids (ABAs), salicylic acid (S.A.), and hydrogen peroxide induce HcHCT expression in Hibiscus cannabinus. HcHCT increases abiotic stress tolerance in plants (Chowdhury et al., 2012). In Cucumis sativus, the HCT expression was reduced with pectinase treatment and directing the phenylpropanoid pathway to generate $\mathrm{H}$-lignin caused p-coumaraldehyde accumulation (Varbanova et al., 2011). HCT is generally a conserved gene family among higher plants (Tohge et al., 2013; Xu et al., 2009). The comprehensive genomewide characterization of HCT gene family as well as focusing on different structural components and functionally active sites would enables us to gain deeper understanding of HCT utilization during specialized metabolism in plants. In the current study, the structural and functional dynamics of the $H C T$ gene family in C. tinctorius was unveiled by conducting genome-wide identification and expression analysis under normal sporadic conditions. This work will also provide unique insights into the underlying regulatory mechanism of plant growth and development under abiotic stress conditions. 


\section{Materials and Methods}

\section{Plant materials and treatment conditions}

The seeds of 'JiHong' No. 1 variety of $C$. tinctorius were purchased from the Tacheng seed company, Xinjiang province of China, and then grown in the experimental station of Jilin Agricultural University under control conditions at $23 \pm 2{ }^{\circ} \mathrm{C}$. The flowering development period in C. tinctorius was recorded approximately 100 days from the date of cultivation. The flower samples from the bud, initial, full, and fade flowering stages were collected on the 99th day, 120th day, 140th day, and 160th day, respectively. In the case of light treatment, the healthy plants of $C$. tinctorius after the initiation of flowering were allowed to grow under the induction of normal light radiation $\left(16.8 \mathrm{MJ} / \mathrm{m}^{2}\right)$ and weak intensity of light irradiation $\left(4.6 \mathrm{MJ} / \mathrm{m}^{2}\right)$ maintained in the experimental station of the laboratory. For MeJA treatment, healthy flowering plants of $C$. tinctorius were treated with $(100 \mu \mathrm{M}$ solution) once daily for 7 days. The flower's petals from each flowering stage were collected and immediately placed in liquid nitrogen and preserved at $-80^{\circ} \mathrm{C}$ until their subsequent use.

\section{Genome-wide identification and sequences retrieval of $\mathrm{Ct} H C T$ s}

The Hidden Markov model (HMMsearch) of the HCT domain (PF02458) at the Pfam database, accessible at http://pfam.xfam.org/ (Finn et al., 2015), was screened to investigate distribution of CtHCTs in the $C$. tinctorius genome. Moreover, we screened the entire set of CtHCT protein sequences for the existence of HXXXD and transferase domains using the online server of MARCOIL available at (http://toolkit.tuebingen.mpg.de/marcoil). The non-redundant protein sequences lacking the two domains of HCT were deleted from the analysis. After the assembly of CtHCT sequences, the genomic and protein sequences of HCTs were collected from Arabidopsis thaliana, Cynara cardaunculus, Helianthus annuus, Lactuca sativa, and Artemisia annuua. The HCTs sequences from $A$. thaliana and other plants were extracted from the Arabidopsis Information Resource (TAIR) (http://www.Arabidopsis.org), NCBI (https://www.ncbi.nlm.nih.gov/), Phytozome (https://phytozome.jgi.doe.gov/pz/portal.htm), and Plantgrn noble (http://plantgrn.noble.org/) respectively. Lastly, a dataset including 82 clean sequences of CtHCTs and 259 members of HCTs from the other five plants were assembled for further bioinformatics analyses. The physicochemical properties of 82 putative $\mathrm{CtHCT}$ proteins were investigated using different online tools. The theoretical isoelectric point (P.I.), protein lengths, and molecular weight (M.W.) of the obtained proteins were analyzed using ExPASyProtParam online tool (available online: at https://web.expasy.org/protparam/). The subcellular localization prediction of each gene was predicted using the cello web server (http://cello.life.nctu.edu.tw) and WoLF PSORT (https://wolfpsort.hgc.jp/).

\section{Phylogenetic reconstruction of HCT proteins}

The full-length amino acid sequences of the $82 \mathrm{CtHCT}$ proteins obtained from the $C$. tinctorius genome were subjected to multiple sequence alignment using Clustal W (2.0). The CtHCT sequences were numbered from CtHCT001-082 following their identification order. To analyse the evolutionary relationship and divergence of CtHCTs, an unrooted neighbour-joining phylogenetic tree with 1000 bootstrap method was generated together with $259 \mathrm{HCT}$ sequences from $A$. thaliana, C. cardaunculus, H. annuus, L. sativa, and A. annuua using MEGA X software version 4.1 (Tamura et al., 2011). The classifications of subfamilies were further analysed for genome-wide comparison.

\section{Analyses of conserved protein motifs and PPI network}

The clear sequences of CtHCTs were added to multiple sequence alignments in Clustal W (2.0) software to investigate the conserved amino acid composition and the presence of conserved protein motifs. The distribution and composition of the conserved protein motifs in CtHCTs were comprehensively investigated by adding each CtHCT protein sequence to MEME web server Version 4.8.1; available at 
http://meme.nbcr.net/meme/cgi-bin/meme.cgi) using the default settings. The logos of these identified motifs were extracted from the MEME server. The graphical representation of protein motifs was edited in EvolView v.2 (http://www.evolgenius.info/). Furthermore, the prediction of protein interactive network of the putative CtHCT proteins was also investigated by uploaded CtHCT sequences to the online web server of STRING database version 10 (https://string-db.org). The hierarchical network of interactor proteins associated with upstream and downstream regulation CtHCTs was created and exported from the STRING database.

\section{Analysis of gene structure, cis-acting units, and Go term enrichment of CtHCTs}

The gene structure organization, including exon and intron and UTR region along with the length of CtHCT genes, was examined from the C.D.sC.D.s and genomic sequences of CtHCT genes with the help of GSDS (Gene Structure Display Server (http://gsds.cbi.pku.edu.cn/index.php) according to the instructions given by (Hu et al., 2014). Furthermore, to investigate the cis-regulatory units of the CtHCT promoter, the $2 \mathrm{~kb}$ upstream 5' UTR flanking sequence of each gene was analyzed using the online webtool of PLACE (https://sogo.dna.affrc.go.jp/). In addition, the G.O. term analysis of C. tinctorius HCTs was determined with the help of Blast2GO software available at (https://www.blast2go.com/) following the instructions given by (Conesa and Götz, 2008). For this purpose, the full-length amino acid residues of CtHCT proteins were added to Blast2Go, and functional annotation of different categories was then identified.

\section{Expression analysis of the putative CtHCTs}

The experimental tissues of flower petals at different stages (bud, initial, fade, and full) were pulverized entirely in liquid nitrogen and then collected into centrifuge tubes. The total RNA extraction was performed using RNA ISOplus reagent (Takara Bio Co., Beijing, China), following the manufacturer's protocol. RNA quality was confirmed using OD260/280 concentrations through NanoDrop 2000 (ThermoFisher Scientific, Beijing, China) and $1 \%$ agarose gel electrophoresis. The first-strand cDNA templates were synthesized from the RNA isolated from each flowering stage using the reverse transcription kit (PrimeScript RT reagent kit with gDNA Eraser (Takara, Japan), following the instruction of the manufacturer's protocols. The quantitative real-time PCR assay was carried out to determine the transcription level of $C t H C T$ genes using SYBR Premix $\mathrm{Ex} \mathrm{Taq}^{\mathrm{m}}$ (TaKaRa). The system of StratageneMx3000P (Stratagene, CA, USA) was utilized to conduct qRTPCR experiments. The relative expression level of CtHCTs at the bud stage was normalized to the housekeeping gene 18s ribosomal RNA expression. The fold change ratio was calculated according to the 2$\Delta \Delta C T$ method (Livak and Schmittgen, 2001). Each experiment was repeated in three independent biological replicates. The gene-specific primers synthesized for each CtHCT are listed in (Table S1).

\section{Statistical analysis}

The results were calculated as mean \pm S.D. with three replicates. The variations between means of each group were assessed by carried through a one-way analysis method of the variance with the help of (Statistix 8.1). P-value equal to 0.05 was kept statistically significant.

\section{Results}

\section{Identification and characterization of CtHCTs in C. tinctorius}

To identify all candidate $H C T$ genes in $C$. tinctorius, we conducted comprehensive searches using the hidden Markov model (HMMsearch) against the C. tinctorius genome. Furthermore, the set of CtHCT sequences were re-investigated for the existence of HXXXD and functional domain of transferase using the online webserver of MARCOIL. Based on the information obtained, a total of 105 HCTs were identified in C. tinctorius genome. Among these, 23 CtHCTs sequences were eliminated from the analysis due to the 
insufficient data and absence of the active HCT functional domains. These $82 C t H C T$ genes were distributed unevenly on different chromosomes, demonstrating the formation of different clusters. The assembly and organization of $C t H C T s$ in $C$. tinctorius revealed that evolutionary events such as tandem duplication and genome repetition might participate in the origin $H C T$ gene family. The $82 \mathrm{CtHCT}$-encoded amino acids were renamed from CtHCT001-CtHCT082. The length of these peptides ranged from 305aa-903aa; molecular weights ranged from $33.88 \mathrm{kDa}$ to $99.69 \mathrm{kDa}$, with an average of $50.40 \mathrm{kDa}$ (Table 1). The isoelectric points ( $\mathrm{pI}$ ) values fall under 5.00 to 9.03 , with an average of 6.51. In addition, the subcellular localization predictions were investigated of which, most of the candidate CtHCTs proteins were localized to the plasma membrane, cytoplasm, nucleus, and mitochondria, respectively. All CtHCTs proteins showed thermal stability due to their aliphatic matching indexes with other globular proteins.

Table 1. Physicochemical properties of CtHCT protein identified in C. tinctorius

\begin{tabular}{|c|c|c|c|c|c|c|c|}
\hline \multirow[b]{2}{*}{ Gene Name } & \multirow[b]{2}{*}{ Gene Ids } & \multicolumn{2}{|c|}{ Physical position } & \multicolumn{3}{|c|}{ Properties of HTC proteins } & \multirow[b]{2}{*}{$\begin{array}{l}\text { Subcellular } \\
\text { localization }\end{array}$} \\
\hline & & $\begin{array}{c}\text { Start } \\
\text { position } \\
\text { (bp) }\end{array}$ & $\begin{array}{c}\text { End } \\
\text { Position } \\
\text { (bp) }\end{array}$ & $\begin{array}{c}\text { Protein } \\
\text { length } \\
\text { (aa) }\end{array}$ & $\mathrm{pI}$ & $\begin{array}{c}\text { Molecular } \\
\text { weight } \\
(\mathrm{kDa})\end{array}$ & \\
\hline CtHCT001 & CCG000516.1 & 746114 & 747439 & 441 & 6.79 & 49.84 & Cytoplasmic \\
\hline CtHCT002 & CCG001378.2 & 5909 & 7267 & 452 & 6.33 & 50.12 & Cytoplasmic \\
\hline CtHCT003 & CCG001379.1 & 24296 & 25537 & 413 & 5.71 & 45.97 & Nuclear \\
\hline CtHCT004 & CCG001803.1 & 211037 & 212986 & 481 & 5.54 & 54.10 & Cytoplasmic \\
\hline CtHCT005 & CCG002140.1 & 229582 & 232205 & 423 & 5.63 & 46.67 & Lysosomal \\
\hline CtHCT006 & CCG002236.2 & 198227 & 202026 & 506 & 5.72 & 56.51 & Cytoplasmic \\
\hline CtHCT007 & CCG002377.1 & 590115 & 591774 & 454 & 6.35 & 50.80 & Mitochondrial \\
\hline CtHCT008 & CCG002378.1 & 600655 & 602022 & 455 & 5.73 & 50.64 & Cytoplasmic \\
\hline CtHCT009 & CCG002482.1 & 863044 & 868400 & 472 & 5.72 & 52.98 & Cytoplasmic \\
\hline CtHCT010 & CCG002510.1 & 97232 & 98599 & 455 & 6.09 & 50.74 & PlasmaMembrane \\
\hline CtHCT011 & CCG003257.1 & 1176385 & 1177676 & 403 & 6.13 & 44.89 & Chloroplast \\
\hline CtHCT012 & CCG003633.1 & 311714 & 313105 & 463 & 5.97 & 51.40 & Cytoplasmic \\
\hline CtHCT013 & CCG003634.1 & 335577 & 336980 & 467 & 5.76 & 51.82 & Cytoplasmic \\
\hline CtHCT014 & CCG003759.1 & 1127462 & 1128874 & 470 & 6.29 & 52.96 & PlasmaMembrane \\
\hline CtHCT015 & CCG003761.1 & 1408445 & 1409809 & 454 & 6.17 & 50.55 & PlasmaMembrane \\
\hline CtHCT016 & CCG003763.1 & 1440050 & 1441414 & 454 & 5.91 & 50.52 & PlasmaMembrane \\
\hline CtHCT017 & CCG003818.1 & 124158 & 126495 & 349 & 6.63 & 39.07 & Cytoplasmic \\
\hline CtHCT018 & CCG003842.1 & 948116 & 950791 & 434 & 6.09 & 47.93 & PlasmaMembrane \\
\hline CtHCT019 & CCG004580.1 & 901107 & 902447 & 446 & 6.49 & 49.88 & Mitochondrial \\
\hline CtHCT020 & CCG004641.1 & 26129 & 27547 & 472 & 5.79 & 52.90 & Plasma Membrane \\
\hline CtHCT021 & CCG004799.1 & 1370991 & 1372205 & 404 & 8.33 & 45.31 & Plasma Membrane \\
\hline CtHCT022 & CCG005260.1 & 27335 & 28777 & 480 & 7.18 & 53.05 & Chloroplast \\
\hline CtHCT023 & CCG005367.1 & 39596 & 40963 & 455 & 5.64 & 50.54 & Plasma Membrane \\
\hline CtHCT024 & CCG005369.1 & 56258 & 57616 & 452 & 6.02 & 50.30 & Plasma Membrane \\
\hline CtHCT025 & CCG006502.1 & 1101963 & 1103285 & 440 & 8.40 & 48.75 & Mitochondrial \\
\hline CtHCT026 & CCG006503.1 & 1110821 & 1112152 & 443 & 7.55 & 48.85 & Cytoplasmic \\
\hline CtHCT027 & CCG006546.1 & 534080 & 536081 & 425 & 5.71 & 47.01 & Cytoplasmic \\
\hline CtHCT028 & CCG007462.1 & 1385829 & 1387238 & 469 & 7.97 & 52.15 & Chloroplast \\
\hline CtHCT029 & CCG007907.1 & 1096935 & 1098290 & 451 & 6.16 & 50.05 & Nuclear \\
\hline CtHCT030 & CCG007909.1 & 1136763 & 1138121 & 452 & 6.20 & 49.86 & Nuclear \\
\hline CtHCT031 & CCG008674.1 & 83325 & 101842 & 465 & 5.71 & 52.11 & Plasma Membrane \\
\hline CtHCT032 & CCG009857.1 & 106340 & 107755 & 443 & 5.98 & 50.01 & Plasma Membrane \\
\hline CtHCT033 & CCG010073.1 & 1973978 & 1975333 & 451 & 6.72 & 50.06 & Mitochondrial \\
\hline CtHCT034 & CCG010534.1 & 2308617 & 2309879 & 420 & 8.59 & 46.96 & Plasma Membrane \\
\hline
\end{tabular}


Fan S et al. (2021). Not Bot Horti Agrobo 49(3):12489

\begin{tabular}{|c|c|c|c|c|c|c|c|}
\hline CtHCT035 & CCG010535.1 & 2313735 & 2314982 & 415 & 5.01 & 45.42 & Chloroplast \\
\hline CtHCT036 & CCG010536.1 & 2321994 & 2323241 & 415 & 7.49 & 46.05 & Plasma Membrane \\
\hline CtHCT037 & CCG010896.1 & 682701 & 685374 & 510 & 8.79 & 56.86 & Plasma Membrane \\
\hline CtHCT038 & CCG012015.1 & 207979 & 209313 & 444 & 5.70 & 49.77 & Cytoplasmic \\
\hline CtHCT039 & CCG012113.1 & 536639 & 537988 & 448 & 6.07 & 49.59 & Plasma Membrane \\
\hline CtHCT040 & CCG012708.1 & 105808 & 107166 & 440 & 8.07 & 49.36 & Nuclear \\
\hline CtHCT041 & CCG012709.1 & 115509 & 116870 & 453 & 7.63 & 50.97 & Nuclear \\
\hline CtHCT042 & CCG012797.1 & 174029 & 182700 & 903 & 5.95 & 99.69 & Nuclear \\
\hline CtHCT043 & CCG014917.1 & 199763 & 201881 & 532 & 7.31 & 59.09 & Chloroplast \\
\hline CtHCT044 & CCG015116.1 & 1991299 & 1992639 & 446 & 6.18 & 50.10 & Cytoplasmic \\
\hline CtHCT045 & CCG015117.1 & 2000394 & 2001758 & 454 & 6.07 & 50.75 & Nuclear \\
\hline CtHCT046 & CCG017040.1 & 626714 & 628000 & 428 & 5.69 & 47.59 & Plasma Membrane \\
\hline CtHCT047 & CCG017170.1 & 163122 & 165066 & 460 & 5.62 & 51.65 & Cytoplasmic \\
\hline CtHCT048 & CCG017171.1 & 169537 & 171323 & 460 & 5.86 & 51.43 & Cytoplasmic \\
\hline CtHCT049 & CCG017720.1 & 26911 & 48638 & 345 & 8.49 & 38.87 & Extracellular \\
\hline CtHCT050 & CCG018048.1 & 11064 & 12464 & 466 & 6.37 & 52.30 & Plasma Membrane \\
\hline CtHCT051 & CCG018068.1 & 325127 & 326672 & 368 & 5.87 & 40.58 & Cytoplasmic \\
\hline CtHCT052 & CCG018070.1 & 334783 & 336192 & 469 & 5.81 & 52.04 & Cytoplasmic \\
\hline CtHCT053 & CCG019384.1 & 387400 & 388767 & 455 & 6.19 & 50.25 & Plasma Membrane \\
\hline CtHCT054 & CCG019474.1 & 81878 & 87176 & 549 & 9.01 & 61.69 & Mitochondrial \\
\hline CtHCT055 & CCG020554.1 & 476616 & 480099 & 462 & 6.61 & 51.58 & Cytoplasmic \\
\hline CtHCT056 & CCG020555.1 & 498145 & 504358 & 461 & 7.10 & 51.02 & Cytoplasmic \\
\hline CtHCT057 & CCG020556.1 & 510109 & 513738 & 461 & 7.57 & 51.28 & Cytoplasmic \\
\hline CtHCT058 & CCG020557.1 & 538870 & 542571 & 462 & 7.57 & 51.19 & Plasma Membrane \\
\hline CtHCT059 & CCG020948.1 & 875842 & 877996 & 556 & 9.03 & 62.40 & Plasma Membrane \\
\hline CtHCT060 & CCG021991.1 & 563097 & 563097 & 411 & 7.69 & 46.50 & Plasma Membrane \\
\hline CtHCT061 & CCG022120.1 & 1791506 & 1795303 & 461 & 6.73 & 51.15 & Mitochondrial \\
\hline CtHCT062 & CCG022233.1 & 165977 & 167374 & 465 & 6.41 & 52.09 & Plasma Membrane \\
\hline CtHCT063 & CCG023912.1 & 2497 & 4184 & 462 & 5.46 & 52.08 & Cytoplasmic \\
\hline CtHCT064 & CCG024342.1 & 1196607 & 1197980 & 440 & 5.39 & 48.19 & Cytoplasmic \\
\hline CtHCT065 & CCG024712.1 & 4710 & 6089 & 459 & 6.38 & 51.50 & Plasma Membrane \\
\hline CtHCT066 & CCG026284.1 & 1500263 & 1501561 & 432 & 5.72 & 47.60 & Chloroplast \\
\hline CtHCT067 & CCG026329.1 & 341504 & 342844 & 446 & 7.93 & 49.34 & Cytoplasmic \\
\hline CtHCT068 & CCG026331.1 & 353669 & 354908 & 380 & 7.05 & 41.79 & Plasma Membrane \\
\hline CtHCT069 & CCG026332.1 & 361244 & 362584 & 446 & 7.94 & 49.42 & Cytoplasmic \\
\hline CtHCT070 & CCG026516.1 & 2255826 & 2258720 & 441 & 6.46 & 48.48 & Plasma Membrane \\
\hline CtHCT071 & CCG027548.1 & 331399 & 333241 & 439 & 5.26 & 48.67 & Cytoplasmic \\
\hline CtHCT072 & CCG027974.1 & 29637 & 31019 & 460 & 6.23 & 50.90 & Plasma Membrane \\
\hline CtHCT073 & CCG028279.1 & 172673 & 174025 & 450 & 5.41 & 49.92 & Cytoplasmic \\
\hline CtHCT074 & CCG028280.1 & 178840 & 180192 & 450 & 8.12 & 49.67 & Cytoplasmic \\
\hline CtHCT075 & CCG028313.1 & 266033 & 267402 & 305 & 6.90 & 33.88 & Chloroplast \\
\hline CtHCT076 & CCG028841.1 & 239752 & 245127 & 430 & 6.67 & 48.16 & Plasma Membrane \\
\hline CtHCT077 & CCG029651.1 & 263479 & 264894 & 471 & 6.03 & 52.98 & Plasma Membrane \\
\hline CtHCT078 & CCG029784.1 & 1533004 & 1534407 & 446 & 5.78 & 50.21 & Cytoplasmic \\
\hline CtHCT079 & CCG029982.1 & 1158515 & 1159906 & 463 & 5.83 & 51.19 & Cytoplasmic \\
\hline CtHCT080 & CCG030854.1 & 299022 & 304069 & 441 & 5.30 & 48.86 & Cytoplasmic \\
\hline CtHCT081 & CCG031372.1 & 30801 & 32141 & 446 & 5.80 & 49.28 & Chloroplast \\
\hline CtHCT082 & CCG031547.1 & 3808 & 4998 & 396 & 5.00 & 44.43 & Plasma Membrane \\
\hline
\end{tabular}




\section{Phylogenetic analysis of $\mathrm{CtHCTs}$}

To further elucidate the evolutionary relationship of the HCT family in C. tinctorius, the protein sequences combined with 259 members of HCTs from A. thaliana, C. cardaunculus, H. annuus, L. sativa, and $A$. annuua were added to the alignments. The selected species were purposely nominated for genome-wide comparison because these plant species shared a relatively high frequency of presenting the $H C T$ gene family across the plant kingdom (Figure S1). A neighbour-joining phylogenetic tree was constructed using the MEGA $\mathrm{X}$ software package with the 1000 bootstrap method. CtHCT family were clustered into six subfamilies designated as the group I, II, III, IV, V, and VI, indicating strong conservation with other species (Figure 1). Comparative phylogenetic studies suggested that most CtHCTs were assembled into group IV with AtHCTs and AuHCTs, which are mainly associated with the function of $\mathrm{r}$ hydroxycinnamates transfer to shikimate during monolignol formation. Similarly, the second most CtHCT containing group was group VI, along with HaHCTs and LsHCTs in abundance, corresponding to the strong conservation of the active sites for carbonyl group and shikimate binding sites. The smallest group was designated as group II containing only one member of CtHCT protein, clustered together with only one CcHCT and two members of AnHCT, suggesting a different catalytic activity and are most likely related to function other than lignin biosynthesis in plants.

Tree scale: 0.1
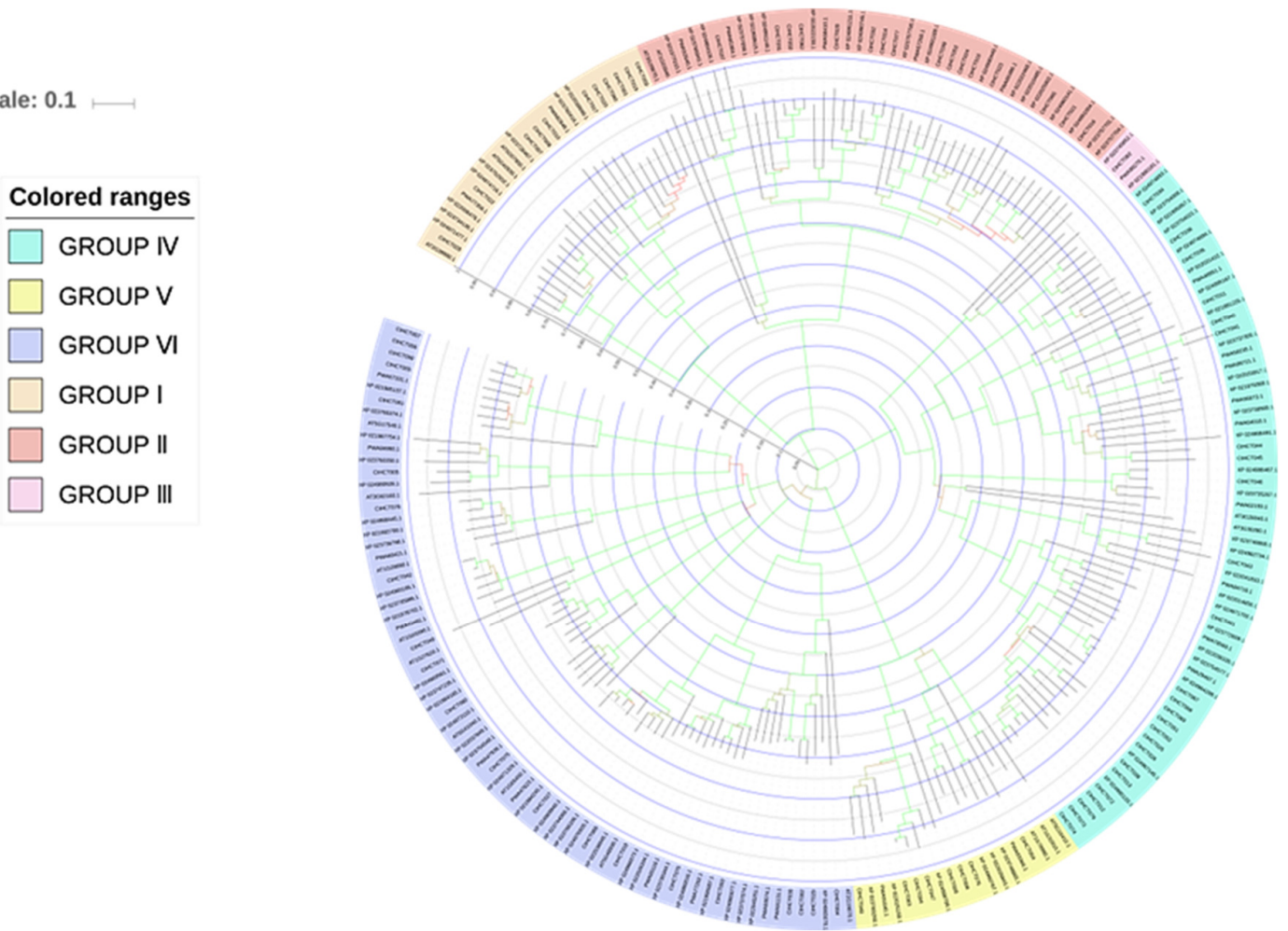

Figure 1. A neighbor-joining phylogenetic tree was generated from the full-length HCT proteins obtained from $A$. thaliana, C. cardaunculus, $H$. annuus, L. sativa, A. annuua, and C. tinctorius

The representation of nodes determines the percentage of bootstrap scores. Different background colours indicated the classification of HCTs from uncommon plant species into different subgroups.

\section{Motifs distribution and protein alignment of $\mathrm{Ct} H C T$ s}

The conserved domains of $\mathrm{CtHCT}$-encoding proteins were identified by aligning the $82 \mathrm{CtHCT}$ amino acid sequences using multiple pairwise alignments. The presence of the two prevalent HCT domains in $C$. tinctorius consisting of HXXXD and DFGWG (Figure S2) were observed. The existence of high-frequency conservation of specified amino acids within the HCT domains suggested crucial hallmarks for the catalytic 
activity of CtHCT in C. tinctorius. Furthermore, the distribution of the conserved motif was screened out with the help of MEME web server with specified settings including the classic mood, selecting the protein standard alphabet, site distribution as zero or one occurrence per sequence, and 10 numbers of motifs. The results confirmed the presence of 10 conserved motifs unevenly organized across CtHCT proteins (Figure 2). For instance, motif $1,3,4,5$, and 7 were found in all subgroups suggesting that these motifs were most frequently conserved in CtHCT proteins.

Moreover, from these findings, we deduced that all closely related CtHCTs proteins clustered together might represent the common composition of these conserved motifs and acquire similar activity. However, motifs 2, 6, 8, 9, and 10 were diversely distributed across all CtHCTs proteins. For example, the occurrence of motif 2 was found absent in the five members of subgroup II, motif 6 were found in all subfamilies except some members of subfamily VI, motif 8 were found nearly in all members of subfamily VI except for subfamily II and IV whereas appeared in only one member of subfamily III, motif 9 were found abundantly in VI but absent in group II, while appeared in only one member of subfamily I and III. Similarly, motif 10 was found unique to group VI and absent in all other subgroups. The full-length logos of these motifs were enlisted in (Figure S3).
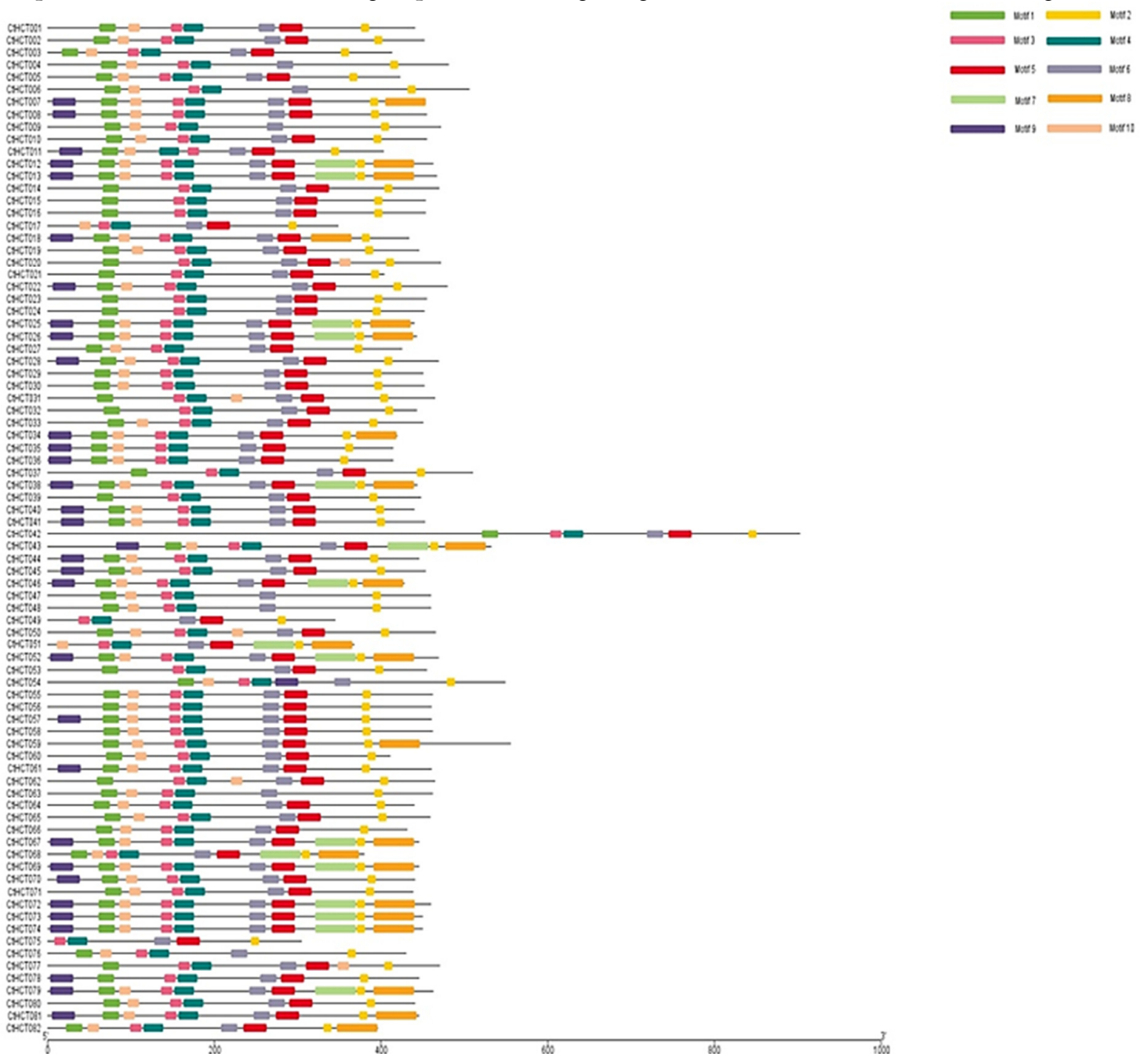

Figure 2. The distribution and organization of the conserved protein motifs found in CtHCT-encoding proteins

The analysis was carried out using the online tool of Multiple EM for Motif Elicitation (MEME version 4.8.1). Different groups of the motif were demonstrated with various colour patterns. The grey line showed the length of each sequence. Each block and colour represent the presence of different conserved motif at a specific location. 


\section{Functional protein interaction network}

The interrelation of CtHCT with other proteins was investigated to link their interaction network involving different biological pathways. Identifying the protein-protein association network could play a fundamental role in predicting the possible function of the putative proteins. A total of 22 interactor proteins were predicted for the CtHCTs as shown in (Figure 3), some of them have already been determined experimentally, such as cinnamate 4-hydroxylase $(\mathrm{C} 4 \mathrm{H})$, p-coumarate 3-hydroxylase $(\mathrm{C} 3 \mathrm{H})$, 4-coumarate: coenzyme A ligase ( $4 \mathrm{CL}$ ), and caffeoyl shikimate esterase (CSE). The role of these interactor proteins was most widely characterized in multiple pathways that occurred in plants during lignin and secondary metabolite biosynthesis. Furthermore, to understand the detailed topology of the interactor proteins with CtHCT proteins, the three-dimensional structures of these proteins were predicted and compared correspondingly determined by their genetically encoded amino acid sequences (Figure S4). As a result, understanding the relationship between 3D arrangements of amino acid sequence and protein structure allows us to draw a significant amount of information for functional prediction of novel protein from genome sequence data and the rational engineering of protein functions. Taken together, the protein-protein interaction network of putative CtHCTs could help us linking crucial biosynthetic pathways and routes leading to specialized metabolism in plants.

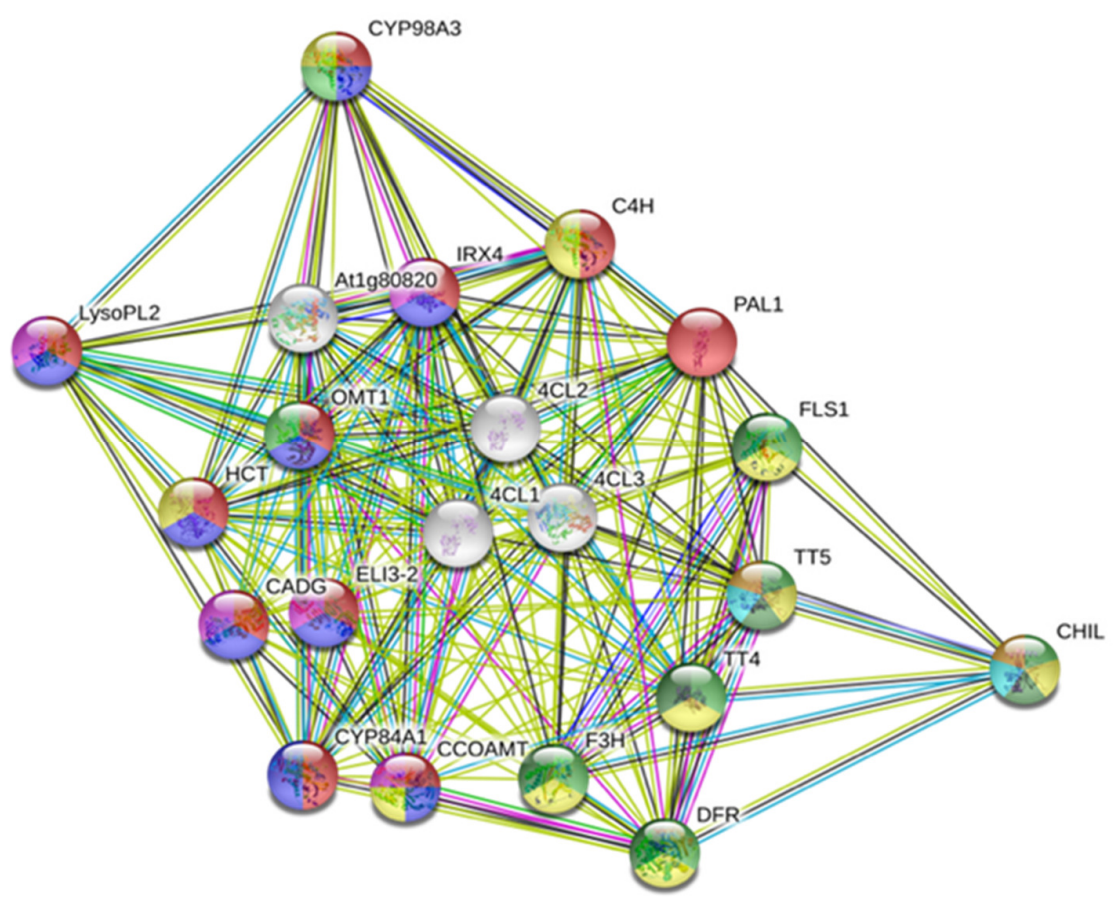

Figure 3. The predictive protein-protein interaction network of putative CtHCT-encoding proteins The most substantial interactions were represented with red, blue, green, yellow, and grey colours. The figure was created with the online database of STRING version 10.

\section{Gene structure organization of CtHCT genes}

The investigation of the intron/exons organization of $C t H C T$ genes was carried out to predict gene structure conservation and investigate their evolutionary relationships using the online GSDS tool. The average length of CtHCT genes ranged between $1000 \mathrm{bp}$ (CtHCT17) to $2675 \mathrm{bp}$ (CtHCT042). The structural organization of each $C t H C T$ gene comprising exons (red), C.D.s (yellow) as well as $5^{\prime}$ and 3 ' UTR regions (blue) is demonstrated in (Figure 4). These results suggested a variable trajectory in exon/intron numbers, C.D.s, and UTR regions were found even in the most closely related members of the same subgroup. For 
example, CtHCT018, CtHCT056, CtHCT057, CtHCT058, and CtHCT070 possess similar gene structures, however, the same members of group 6 contains different number of exons and introns including CtHCT002, CtHCT005, CtHCT049, CtHCT064, CtHCT066, and CtHCT078. The diversity in gene structures of $C t H C T$ genes indicated multiple evolutionary mechanisms such as gene recombination, gene duplication, alternative splicing, and transposon, resulting in new gene structures and transcripts that form unique polypeptides with different biological functions.

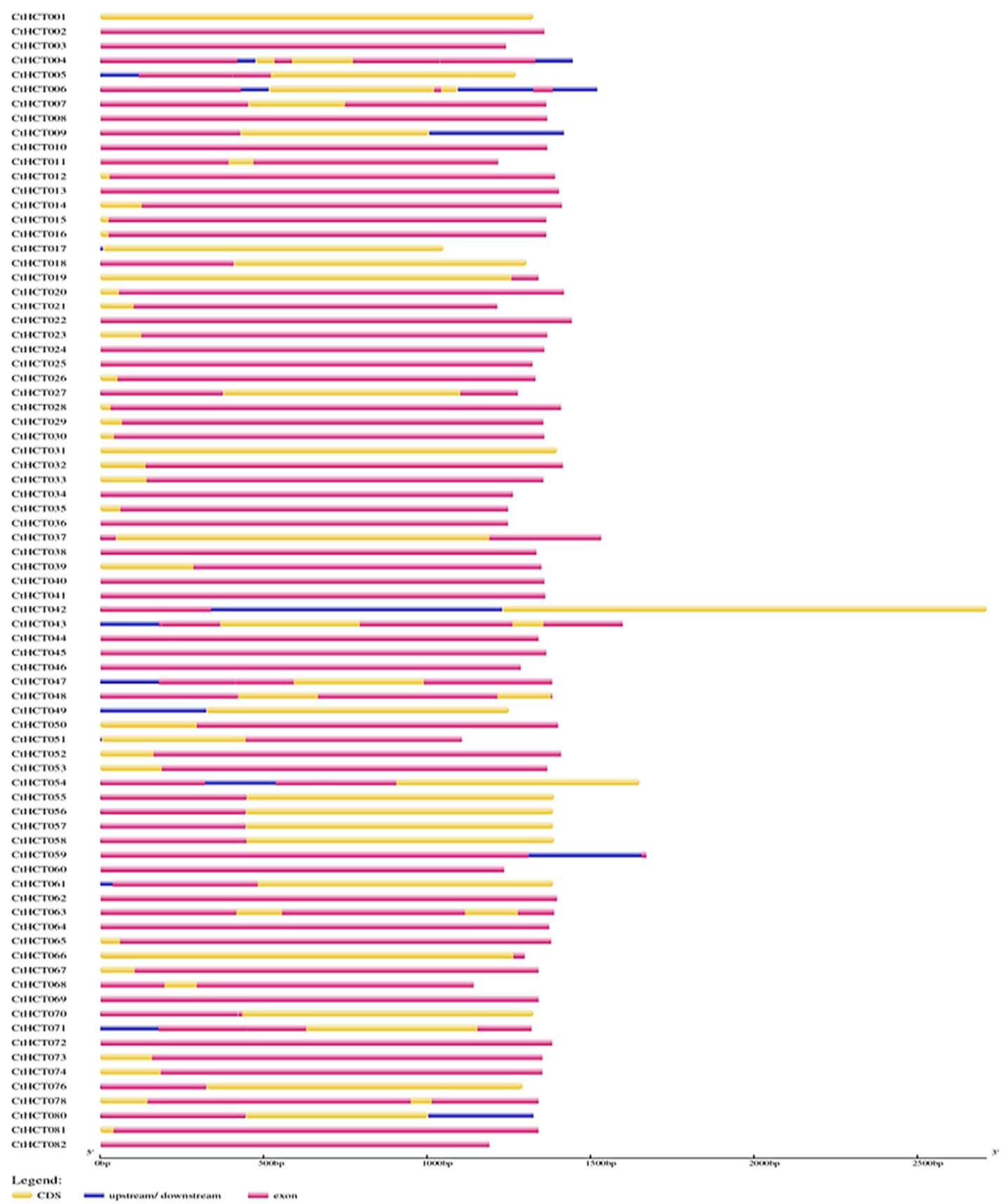

Figure 4. Gene structure organization of $C t H C T$ genes

Different elements of the gene structure, including (5' and $3^{\prime}$ UTR, Exons and CDS) of 82 putative HCT genes of $C$. tinctorius were crafted with the Gene Structure Display Server (GSDS; 2.0). 


\section{Cis-regulatory units of $\mathrm{CtHCT}$ genes}

To explore the functional diversity and regulatory system of the CtHCT gene family, we investigated cis-regulatory elements within the promoter region of each gene. A total of 20 frequent cis-regulatory units were identified in the $2000 \mathrm{bp}$ genomic sequences located upstream from the initiation codon or 5 ' untranslated region (5'UTR) of CtHCT genes. Most of the cis-elements found abundantly in the promoter region of $C t H C T$ genes include hormonal responsive elements, particularly gibberellins, jasmonic acid, salicylic acid, abscisic acid, and auxin-responsive elements. Furthermore, diverse group of regulatory units related to tissue-specific expression were also detected, such as meristems, endosperm, root, and seed-specific regulatory units. Apart from that, various defense and abiotic stress-associated responsive elements, such as light and low temperature-responsive elements combined with cell cycle regulatory units and metabolic-related responsive units were also detected in the promoter region of $C t H C T$ genes of $C$. tinctorius (Figure S5). These findings revealed the flexibility and functional diversity of $C t H C T$ genes involving their potential roles in specialized metabolism and diverse biological activities in plants.

\section{Functional annotation of CtHCT genes}

The G.O. term analysis was performed to assign functional annotation to putative $C t C H C T$-encoding genes. All CtHCTs were divided into three functional categories, including biological processes (B.P.), molecular function (M.F.), and cellular component (CC). A bulk of CtHCT genes were enriched into biological processes term followed by molecular function and cellular component. The most enriched G.O. terms of biological processes contain biosynthetic, metabolic, and cellular processes which include biotic and abiotic stimuli, defense responses, cell wall organization and biogenesis, cellulose metabolic, and biosynthetic processes (Figure S6). In the molecular function term, the top-ranked G.O. terms of binding and catalytic activities were enriched which include enzyme inhibitor and regulator activity, molecular function regulator, copper ion binding, protein histidine kinase activity, carboxylic ester hydrolase activity, and pectinesterase activity. The most enriched G.O. terms such as the cell wall, external encapsulating structure, cell periphery, and respiratory chain were assigned to the cellular component category (Figure S6). These results emphasized the potential roles of $C t H C T s$ in a variety of biosynthetic and crucial metabolic processes which may directly or otherwise participate in regulating plant responses against external stimuli.

\section{HCT expression profiles at different flowering stages of C. tinctorius}

The digital expression level of $C t H C T$ genes was primarily calculated with FPKM (fragments per $\mathrm{Kb}$ per million reads) statistics of each $C t H C T$ gene using the software package of featureCounts (v1.5,0-p3). The data was obtained from the whole transcriptome shotgun sequencing in four different flowering stages of $C$. tinctorius, including bud, initial, full, and fade stages. The data have been deposited in the public database of NCBI under the accession number (PRJNA399628). As described in (Figure S7), the expression level of $C t H C T$ genes was divided into different cluster groups demonstrating the differential expression pattern of these transcripts at four flowering stages in $C$. tinctorius. Furthermore, to validate the biological expression level of $C t H C T$ transcripts, we performed the qRT-PCR analysis of 20 genes in four different flowering stages of $C$. tinctorius (bud, initial, full, and fade). Expectedly, the expression patterns of these $C t H C T$ genes across

all flowering stages were found consistent with RNA-seq results. For instance, the expression trend of CtHCT005, CtHCT019, CtHCT030, CtHCT055, CtHCT077 and CtHCTO81 was increased upwards at the initial and full phase of flower development (Figure 5). Similarly, the transcript abundance of the CtHCT009, CtHCT028, CtHCT031 candidate genes was exhibited at the full and fade flowering phase. However, the relative transcript abundance of CtHCTOO1, CtHCTO28, CtHCT033, and CtHCTO48 was detected only at the full flowering phase indicating their transcriptional regulation full bloom flowering period of C. tinctorius. On the contrary, the relative fold expression level of CtHCTO17, CtHCT027, CtHCT031, CtHCT039, CtHCTO40 and CtHCT059 was peaked at the fading stage of flower development (Figure 5). 
Mostly, the $C t H C T$ genes expression level in fading stages of flower development was four-fold higher than the rest of the three stages. The expression preference of $C t H C T$ genes in different flowering phases suggested a significant correlation with flower developmental and regulation of secondary metabolism in plants.

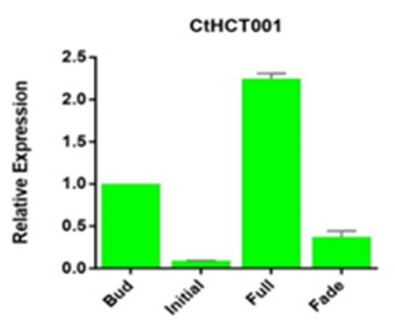

CtHCT019

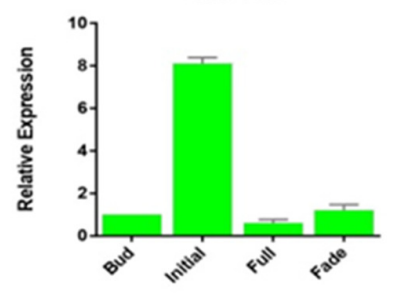

CtHCT031

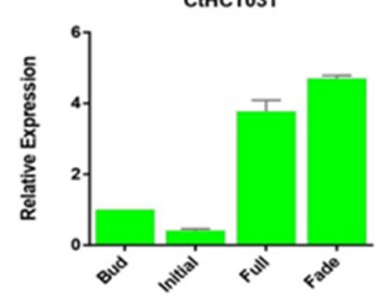

CtHCT040

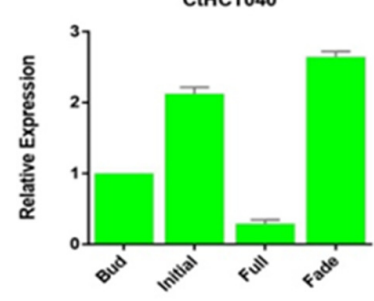

CtHCT065

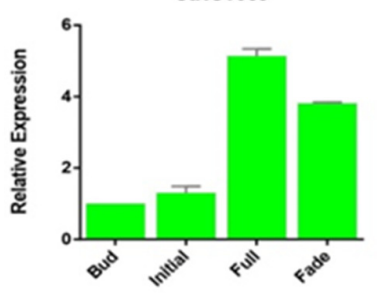

CtHCT005

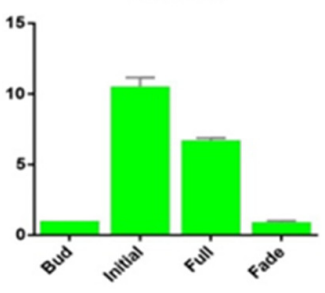

CtHCT027

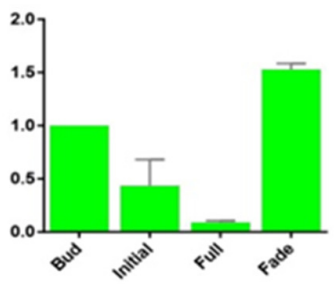

CtHCT033

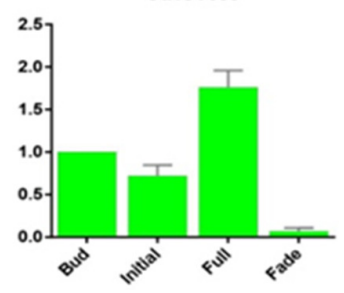

CtHCT048

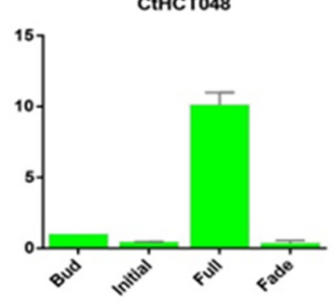

CtHCT069

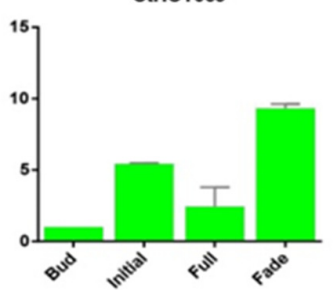

CtHCT009

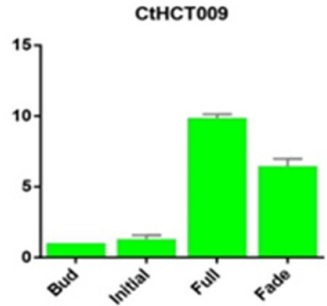

CtHCT028

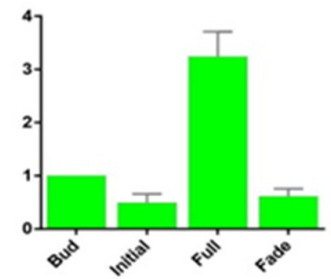

CtHCT036

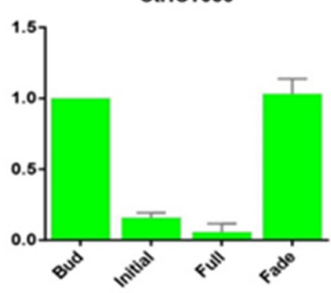

CtHCT055

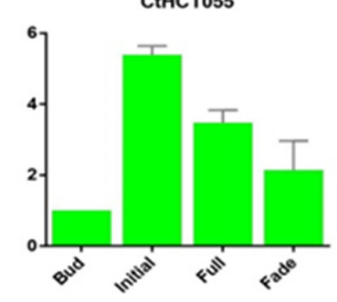

CtHCT077

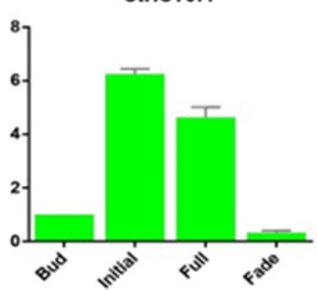

CtHCT017

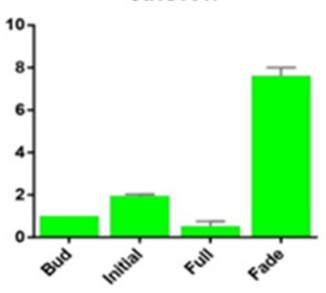

Ctнст030

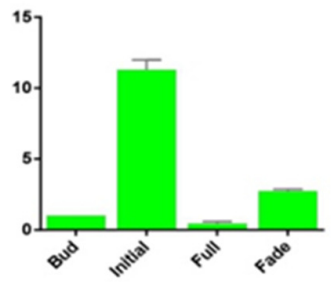

CtHCT039

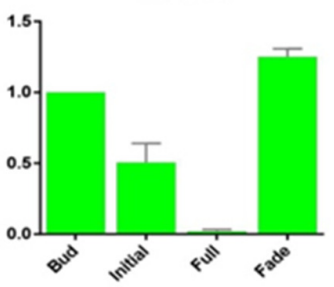

CtHCT059

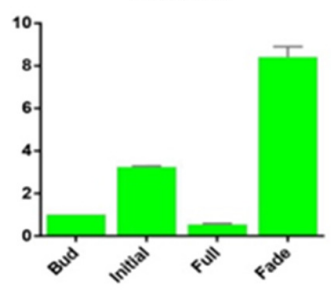

CtHCT081

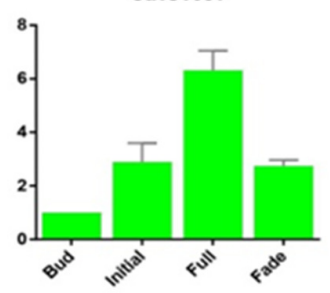

Figure 5. Expression profiling of HCT genes in C. tinctorius at four different flower developmental stages, including bud, initial, full, and fade

The 18 s ribosomal RNA genes were used as a housekeeping gene in our analysis. The data was calculated using the 2$\Delta \Delta^{\mathrm{CT}}$ method. 


\section{Expression profiling of $\mathrm{CtHCT}$ genes under light irradiation}

Here we analyzed the expression profiling of CtHCT genes at four different phases of flower development in $C$. tinctorius in response to normal and low light irradiation using the qRT-PCR assay. Following weak light irradiation $\left(4.6 \mathrm{MJ} / \mathrm{m}^{2}\right)$, the transcription level of selected $C t H C T$ genes was significantly induced through all flowering stages under investigation. However, the expression trend was found variable for each transcript than the normal light irradiation condition $\left(16.8 \mathrm{MJ} / \mathrm{m}^{2}\right)$. Following weak light irradiation at bud flowering stage, the expression level of the most of CtHCT genes including CtHCT017, CtHCT019, CtHCT030, CtHCT017, CtHCT039, CtHCT048, CtHCT055, CtHCT065, and $C t H C T 081$ was significantly up-regulated indicating different folds of transcript abundance. However, CtHCT001, CtHCT033, and CtHCT059 expression levels were down-regulated at the bud flowering stage (Figure 6).
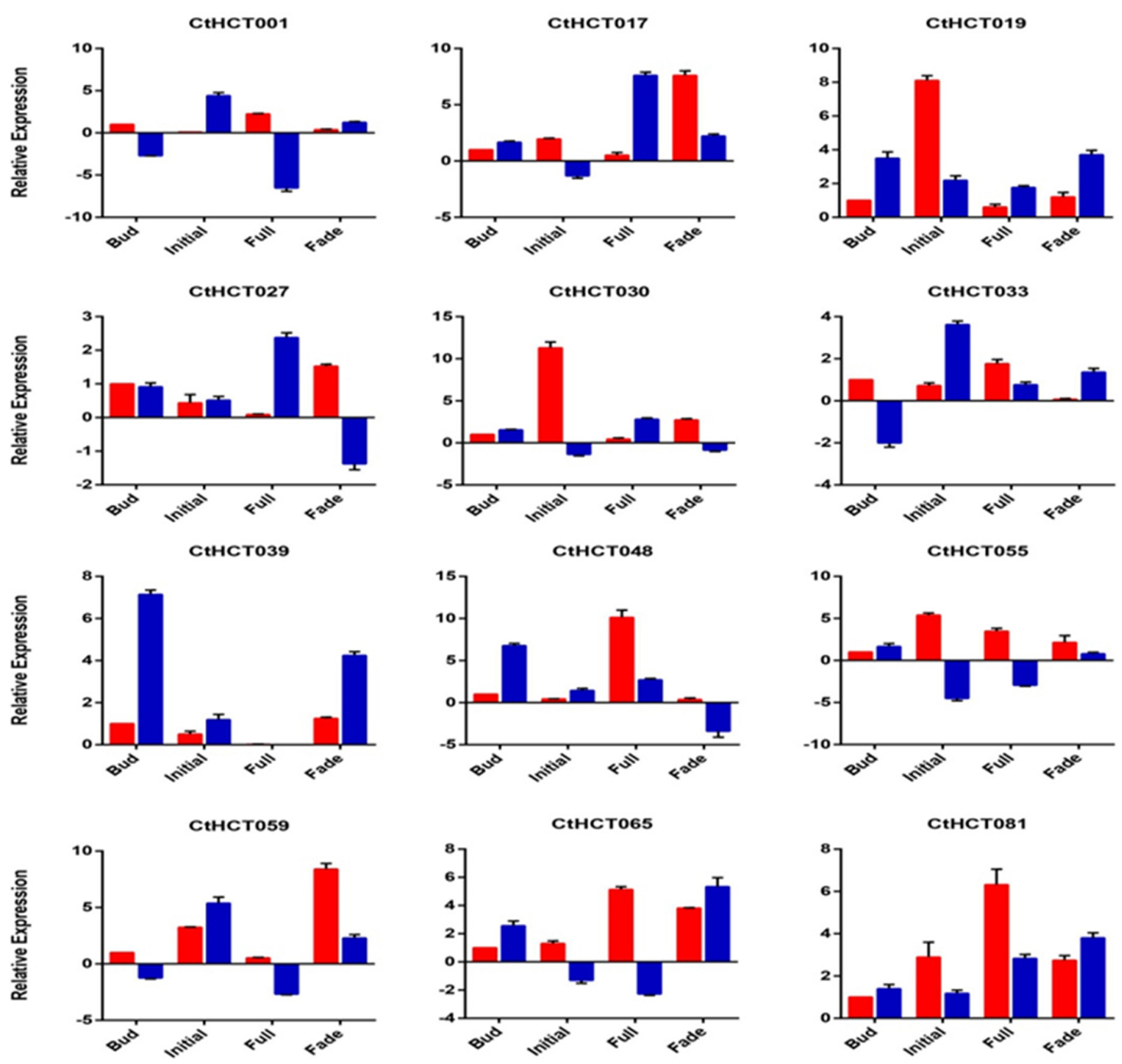

Figure 6. Expression profiling of $C t H C T$ genes at four different flowering stages of $C$. tinctorius under light irradiation using qRT-PCR analysis

The red bars represent the control treatment group under normal light irradiation $\left(16.8 \mathrm{MJ} / \mathrm{m}^{2}\right)$, whereas blue bars denote the treatment group induced with $\left(4.6 \mathrm{MJ} / \mathrm{m}^{2}\right)$. The $18 \mathrm{~s}$ ribosomal RNA genes were used as the housekeeping gene. The data was calculated using the $2-\Delta \Delta^{\mathrm{CT}}$ method. 
Moreover, after the induction of weak light irradiation at the initial flowering phase of $C$. tinctorius, the transcription level of CtHCT001, CtHCT033, CtHCT039, CtHCT048, CtHCT059 was increased up to 2-3 folds, whereas the expression level was declined in case of CtHCTO17, CtHCTO19, CtHCT030, CtHCT055, CtHCT065, and CtHCT081 genes.Under the same light intensity at the full flowering stage, the expression level of the most CtHCT genes showed a downwards trend, including in CtHCTOO1, CtHCT033, CtHCTO48, CtHCT055, CtHCT059, and CtHCT065; however, the expression level was induced upwards in CtHCT017, CtHCT019, CtHCT027, and CtHCT030. During fading stage, the expression of CtHCT genes such as CtHCT001, CtHCT019, CtHCT033, CtHCT039, CtHCT065, and CtHCT081 was up-regulated under weak light induction, whereas the expression pattern was declined in case of CtHCT017, CtHCT027, CtHCT030, CtHCT048, CtHCT055, and CtHCT059 (Figure 6). These findings of the of $C t H C T$ genes under weak light irradiation suggested positive insights into understanding the regulation of stress responses by activating their genetic machinery in combination with other possible factors that interconnect early stress responsive mechanisms.

\section{Expression profiling of CtHCT genes under MeJA treatment}

Methyl jasmonate is an important hormone involved in regulating various growth and defense-related signaling pathways in plants. Herein, the expression level of $C t H C T$ genes under the MeJA treatment was investigated using qRT-PCR analysis. Following the results of qRT-PCR, a variable expression profile of $C t H C T$ genes under MeJA stress was detected than the normal condition. Among $10 C t H C T$ genes, the expression level of CtHCT001, CtHCT048, and CtHCT081 genes respectively up-regulated up to 2 folds at bud flowering and 2-4 folds at initial flowering under MeJA stress (Figure 7). Similarly, in combination with bud and initial flowering, the transcript abundance of CtHCT059also suggested an increased expression trend with a slight change displaying up-regulation at the full flowering stage. In addition, the expression level of CtHCT017, CtHCT039, and CtHCT065 following induction with MeJA rises at both the initial and full phases of flower development. In contrast, the expression was down-regulated in other flowering parts. In contrast, the transcription of CtHCTO19 and CtHCTO3O under the same treatment of MeJA demonstrated an upward expression trend at the bud and complete flowering stages. It was down-regulated at the remaining flowering stages (Figure 7). Interestingly, the expression level of CtHCT055 was down-regulated at all

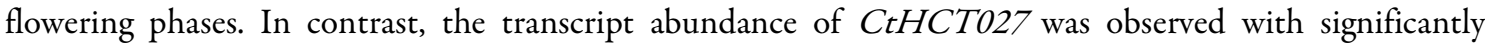
increased expression patterns at the full and fading stages of flower development. Based on the variable expression network of $\mathrm{CtHCT}$ genes under MeJA induction at different stages of flower transitions, it is suggested that the transcription level of $C t H C T s$ may play a pivotal role in facilitating the adaptation of $C$. tinctorius in fluctuating environments. 

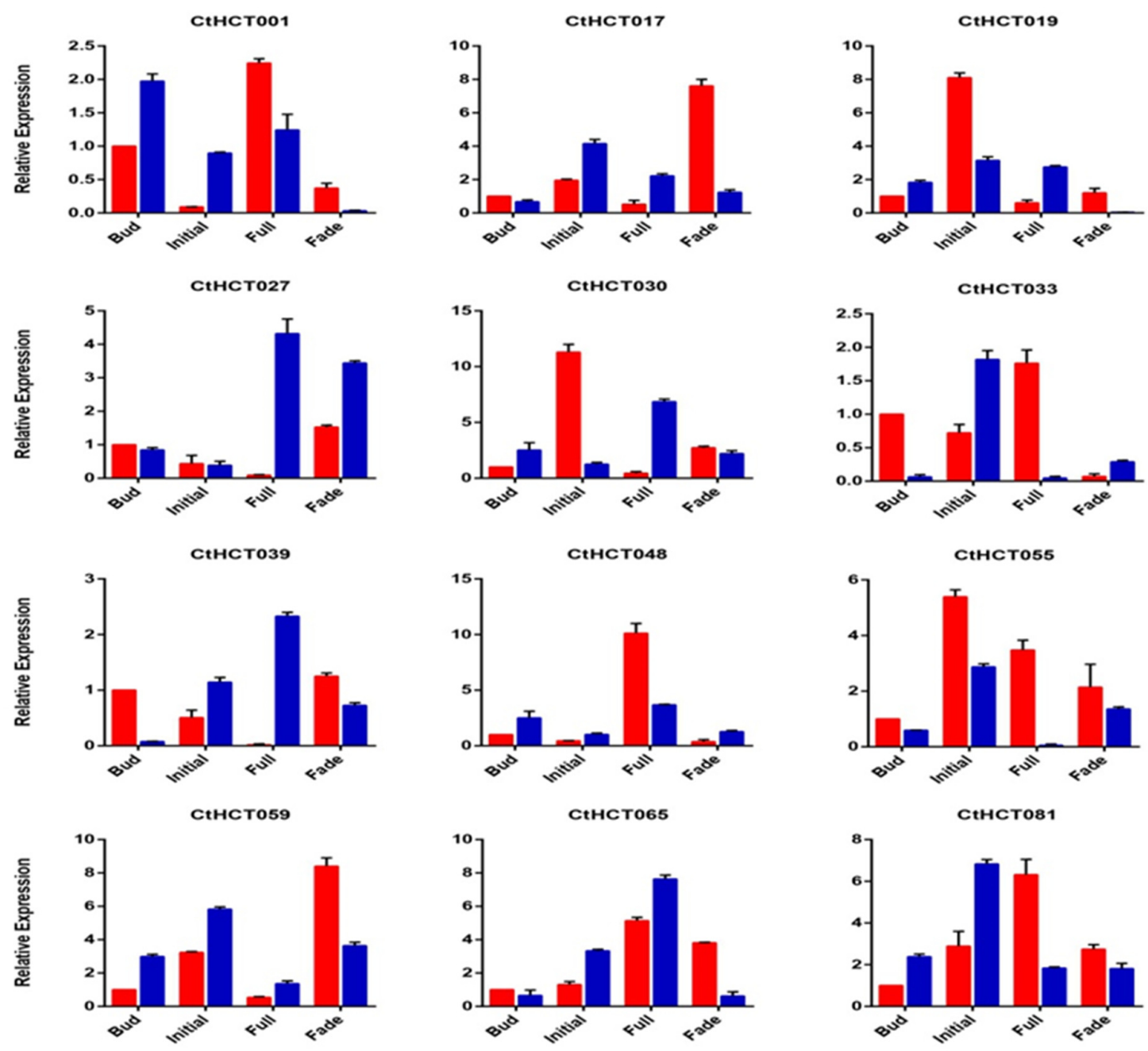

Figure 7. Expression profiling of $C t H C T$ genes at four different flowering stages of $C$. tinctorius under methyl jasmonate treatment using qRT-PCR analysis

The red bars represent the control treatment group (no treatment), whereas the blue bars denote the treatment group induced with MeJA $(100 \mu \mathrm{M})$. The 18 s ribosomal RNA genes were used as a housekeeping gene. The data was calculated using the $2-\Delta \Delta^{\mathrm{CT}}$ method.

\section{Discussion}

The HCT gene family is mainly involved during the regulatory mechanism of monolignol biosynthesis in different plant species (Shadle et al., 2007; Sun et al., 2018). Here, we extensively characterized the HCT gene family in $C$. tinctorius and provided a complete genome-wide overview of these genes alongside their structural and functional active sites interlinked with the regulation of abiotic stress responses in plants. In general, the assembly of the HCT gene family of $C$. tinctorius in comparison to other plants shared variable size in the total genome, including Arabidopsis (Initiative, 2000), strawberry (Shulaev et al., 2011), pear (Wu et al., 2013), apple (Velasco et al., 2010), peach (Verde et al., 2013). Further comparative analysis revealed that the occurrence of conserved amino acids at specific positions in $C t H C T$ was found consistent with the Arabidopsis (D'Auria, 2006) and pear HCTs (Ma et al., 2017). Moreover, the existence of other conserved amino acid residues indicating more than $60 \%$ similarity was consistent with Populus nigra (Vanholme et al., 
2013) and Coffeaca nephora (Lepelley et al., 2007). These findings suggested that the high frequency of the conserved amino acid residues could be crucial for identifying putative function of $C t H C T$ genes.

The investigation of the cis-elements in the promoter region of $C t H C T$ genes revealed various critical regulatory units involved during the counter-response of plants against different abiotic stressors. The occurrence of these top-ranked abiotic stress-responsive elements in $C t H C T$ genes includes abscisic acid, low temperatures, gibberellins, jasmonic acid, salicylic acid, auxin, defense and stress-responsive factors, and endosperm-specific expression. As found by (Dang et al., 2011), these types of cis-regulatory elements were explicitly identified in pea plants against abiotic stress responses. In agreement with these findings, the results of of cis-elements of CtHCT genes also suggested important hallmarks involving plant adaptation to various abiotic stresses and signal transduction of hormones during plant growth and development. In addition to cisregulatory elements, the enrichment of the $H C T$-encoding proteins in the core pathway of phenylpropanoid biosynthesis and other essential classes of secondary metabolites have been described in several plants, such as Linum usitatissimum (Tripathi and Agrawal, 2013), tobacco (Tamagnone et al., 1998), and Eucalyptus globules (Shinya et al., 2014). In this study, we also investigated that most of the CtHCT-encoding proteins were enriched in biosynthetic, metabolic, and cellular processes containing responses to biotic and abiotic stimuli, defense responses, cell wall organization and biogenesis, cellulose metabolic and biosynthetic processes, and external encapsulating structure organization. These findings strongly highlight essential clues into the putative role of $C t H C T$ genes in secondary metabolism and understanding the abiotic stress resistance mechanism in C. tinctorius.

HCT genes played a critical role in plant growth and development-related activities. For example, the HCT1 and HCT2 of red clover plants were expressed in all tissues, including stems, leaves, and flowers, but was found higher in flowers than expression in unexpanded leaves, mature leaves, and stems (Sullivan, 2009). Similarly, Populus trichocarpa possesses seven PtrHCTs that can be expressed in the tissues of various plant parts and exhibit differences concerning their relative performance. In particular, PtrHCT1 and PtrHCT6are primarily expressed in stem tissues, whereas PtrHCT3 has a higher expression level in leaf tissues (Shi et al., 2009). Another study revealed that the expression pattern of the HcHCT transcript was ubiquitous in all parts of a 4-week-old plant but was relatively high in roots and mature flowers. The highest $H c H C T$ transcript was detected in young flowers and young leaves during flowering and leaf development (Chowdhury et al., 2012). HcHCT showed high expression levels in flowers and roots, suggesting that HcHCT participates in the biosynthesis of secondary metabolites in floral and root tissues. Given the previous studies, the diverse expression profiling of $C t H C T$ genes at four different flowering stages of $C$. tinctorius was also detected. The transcription regulation and expression preference of $C t H C T$ genes at various flowering stages indicated a significant correlation of these transcripts with plant growth and development and regulation of secondary metabolism.

Furthermore, the natural exposure of plants to various abiotic and biotic stresses leads to generating several mechanisms in cell wall modification to protect themselves against these stresses. The potential of lignin to protects cell wall degradation by maintaining its polysaccharides level against pathogenic microbes and stressinduced degradation. Lignin act as an antioxidant in a plant, encountering heat stress and eventually increase plant tolerance against various stress conditions (Bhardwaj et al., 2014). As a point of importance, the optimum light intensity and temperature required for germination and average growth of $C$. tinctorius are $\left(16.8 \mathrm{MJ} / \mathrm{m}^{2}\right)$ and $35^{\circ} \mathrm{C}$ (Torabi et al., 2016). Fluctuation in light intensity and temperature can affect the physiological and development activities of $C$. tinctorius (Torabi et al., 2016). In addition, MeJA treatment also demonstrated similar results in Norway spruce where the HCT genes showed induced expression level (Chowdhury et al., 2012). The regulatory mechanism of abiotic stress tolerance in $C$. tinctorius by conducting the expression profiling of $C t H C T$ genes under low light irradiation $\left(4.6 \mathrm{MJ} / \mathrm{m}^{2}\right)$ and MeJA stress using qRT-PCR analysis was also examined. These results demonstrated that $C t H C T$ transcription shared a diverse expression pattern through different flower development stages in C. tinctorius following induction with weak light irradiation and MeJA treatment. These data suggested that the up-regulation and down-regulation of CtHCT genes at 
certain stages of flower development could be involved in the defense-related pathways in coordination with J.A. signalling pathways (Chowdhury et al., 2012) that define early stress response to specific and broadspectrum stress tolerance mechanisms. However, more efforts are still needed to elucidate the explicit functional role of $C t H C T$ genes in C. tinctorius.

\section{Conclusions}

This study provides the first comprehensive genome-wide analysis explaining various structural and functional components of $C t H C T$ genes. From these findings, it was revealed that various conserved entities such as protein motifs, cis-acting elements, gene structure, and functional enrichments could be crucial in predicting the function of $C t H C T$ genes during plant growth and development. In addition, a group of CtHCT genes showed preferential expression in developing flowers of $C$. tinctorius under normal and abiotic stress conditions, suggesting the regulation of stress responsive mechanism in flower tissues. Together, these findings could pave the wave for the discovery of key genes involved in lignin biosynthesis and the foundation for engineering C. tinctorius with enhanced lignin content.

\section{Authors' Contributions}

Conceptualization, NA, and LX; Data curation, NH and WN; Formal analysis, MX; Methodology, SF; Software, ZX and YZ; Supervision, LX, JL and YN; Validation, SF and NA; Writing - original draft, SF and NA; Writing - review \& editing, NA and AM. All authors read and approved the final manuscript.

\section{Acknowledgments}

This work was supported by the National Natural Science Foundation of China (No. 31771868, 31501366), the Science and Technology Development Project of Jilin Province (No. 20190201172JC,20190201175JC,20150623024TC-11), Basic Agricultural Science and Technology Program of Wenzhou Science \& Technology Bureau (No. N20190006), and the student innovation and entrepreneurship training program of Jilin Agricultural University.

\section{Conflict of Interests}

The authors declare that there are no conflicts of interest related to this article.

\section{References}

Bhardwaj R, Handa N, Sharma R, Kaur H, Kohli S, Kumar V, Kaur P (2014). Lignins and abiotic stress: an overview. Physiological Mechanisms and Adaptation Strategies in Plants Under Changing Environment. Springer, pp 267296. https://doi.org/10.1007/978-1-4614-8591-9_10

Chao N, Qi Q, Li S, Ruan B, Jiang X, Gai Y (2021). Characterization and functional analysis of the HydroxycinnamoylCoA: shikimate hydroxycinnamoyl transferase (HCT) gene family in poplar. PeerJ 9:e10741.

Chiang YC, Levsh O, Lam CK, Weng JK, Wang Y (2018). Structural and dynamic basis of substrate permissiveness in hydroxycinnamoyltransferase (HCT). PLoS Computational Biology 14(10):e1006511. https://doi.org/10.1371/journal.pcbi.1006511 
Chowdhury EM, Choi BS, Park SU, Lim HS, Bae H (2012). Transcriptional analysis of hydroxycinnamoyl transferase (HCT) in various tissues of Hibiscus cannabinus in response to abiotic stress conditions. Plant Omics 5(3):305.

Conesa A, Götz S (2008). Blast2GO: A comprehensive suite for functional analysis in plant genomics. International Journal of Plant Genomics 2008:619832. https://doi.org/10.1155/2008/619832

D'Auria JC (2006). Acyltransferases in plants: a good time to be BAHD. Current Opinion in Plant Biology 9(3):331340. https://doi.org/10.1016/j.pbi.2006.03.016

Dang HQ, Tran NQ, Tuteja R, Tuteja N (2011). Promoter of a salinity and cold stress-induced MCM6 DNA helicase from pea. Plant Signaling and Behavior 6(7):1006-1008. https://doi.org/10.4161/psb.6.7.15502

Escamilla-Treviño LL, Shen H, Hernandez T, Yin Y, Xu Y, Dixon RA (2014). Early lignin pathway enzymes and routes to chlorogenic acid in switchgrass (Panicum virgatum L.). Plant Molecular Biology 84(4-5):565-576. https://doi.org/10.1007/s11103-013-0152-y

Eudes A, Pereira JH, Yogiswara S, Wang G, Teixeira Benites V, Baidoo EE, ... Loqué D (2016). Exploiting the substrate promiscuity of hydroxycinnamoyl-CoA: shikimate hydroxycinnamoyl transferase to reduce lignin. Plant and Cell Physiology 57(3):568-579. https://doi.org/10.1093/pcp/pcw016

Finn RD, Coggill P, Eberhardt RY, Eddy SR, Mistry J, Mitchell AL, ... Sangrador-Vegas A (2015). The Pfam protein families database: towards a more sustainable future. Nucleic Acids Research 44(D1):D279-D285. https://doi.org/10.1093/nar/gkv1344

Hu B, Jin J, Guo AY, Zhang H, Luo J, Gao G (2014). GSDS 2.0: an upgraded gene features visualization server. Bioinformatics 31(8):1296-1297. https://doi.org/10.1093/bioinformatics/btu817

Initiative AG (2000). Analysis of the genome sequence of the flowering plant Arabidopsis thaliana. Nature 408(6814):796. https://doi.org/10.1038/35048692

Lepelley M, Cheminade G, Tremillon N, Simkin A, Caillet V, McCarthy J (2007). Chlorogenic acid synthesis in coffee: An analysis of CGA content and real-time RT-PCR expression of HCT, HQT, C3H1, and CCoAOMT1 genes during grain development in C. canephora. Plant Science 172(5):978-996. https://doi.org/10.1016/j.plantsci.2007.02.004

Livak KJ, Schmittgen TD (2001). Analysis of relative gene expression data using real-time quantitative PCR and the 2$\Delta \Delta$ CT method. Methods 25(4):402-408. https://doi.org/10.1006/meth.2001.1262

Ma C, Zhang H, Li J, Tao S, Qiao X, Korban SS, Zhang S, Wu J (2017). Genome-wide analysis and characterization of molecular evolution of the HCT gene family in pear (Pyrus bretschneideri). Plant Systematics and Evolution 303(1):71-90. https://doi.org/10.1007/s00606-016-1353-z

Payyavula RS, Shakya R, Sengoda VG, Munyaneza JE, Swamy P, Navarre DA (2015). Synthesis and regulation of chlorogenic acid in potato: Rerouting phenylpropanoid flux in HQT-silenced lines. Plant Biotechnology Journal 13(4):551-564. https://doi.org/10.1111/pbi.12280

Roh JS, Han JY, Kim, JH, Hwang JK (2004). Inhibitory effects of active compounds isolated from safflower (Carthamus tinctorius L.) seeds for melanogenesis. Biological and Pharmaceutical Bulletin 27(12):1976-1978. https://doi.org/10.1248/bpb.27.1976

Shadle G, Chen F, Reddy MS, Jackson L, Nakashima J, Dixon RA (2007). Down-regulation of hydroxycinnamoyl CoA: shikimate hydroxycinnamoyl transferase in transgenic alfalfa affects lignification, development and forage quality. Phytochemistry 68(11):1521-1529.

Shi R, Sun YH, Li Q, Heber S, Sederoff R, Chiang VL (2009). Towards a systems approach for lignin biosynthesis in Populus trichocarpa: transcript abundance and specificity of the monolignol biosynthetic genes. Plant and Cell Physiology 51(1):144-163. https://doi.org/10.1093/pcp/pcp175

Shinya T, Hayashi K, Onogi S, Kawaoka A (2014). Transcript level analysis of lignin and flavonoid biosynthesis related genes in Eucalyptus globulus. American Journal of Plant Sciences 5(18):2764. https://doi.org/10.4236/ajps.2014.518293

Shulaev V, Sargent DJ, Crowhurst RN, Mockler TC, Folkerts O, Delcher AL, ... Mane SP (2011). The genome of woodland strawberry (Fragaria vesca). Nature Genetics 43(2):109. https://doi.org/10.1038/ng.740

Sonnante G, D’Amore R, Blanco E, Pierri CL, De Palma M, Luo J, ... Martin C (2010). Novel hydroxycinnamoylcoenzyme A quinate transferase genes from artichoke are involved in the synthesis of chlorogenic acid. Plant Physiology 153(3):1224-1238. https://doi.org/10.1104/pp.109.150144

Sullivan M (2009). A novel red clover hydroxycinnamoyl transferase has enzymatic activities consistent with a role in phaselic acid biosynthesis. Plant Physiology 150(4):1866-1879. https://doi.org/10.1104/pp.109.136689 
Sun CH, Yang CY, Tzen JT (2018). Molecular identification and characterization of hydroxycinnamoyl transferase in tea plants (Camellia sinensis L.). International Journal of Molecular Sciences 19(12):3938. https://doi.org/10.3390/ijms19123938

Tamagnone L, Merida A, Parr A, Mackay S, Culianez-Macia FA, Roberts K, Martin C (1998). The AmMYB308 and AmMYB330 transcription factors from Antirrhinum regulate phenylpropanoid and lignin biosynthesis in transgenic tobacco. The Plant Cell 10(2):135-154. https://doi.org/10.1105/tpc.10.2.135

Tamura K, Peterson D, Peterson N, Stecher G, Nei M, Kumar S (2011). MEGA5: molecular evolutionary genetics analysis using maximum likelihood, evolutionary distance, and maximum parsimony methods. Molecular Biology and Evolution 28(10):2731-2739. https://doi.org/10.1093/molbev/msr121

Tohge T, Watanabe M, Hoefgen R, Fernie AR (2013). The evolution of phenylpropanoid metabolism in the green lineage. Critical Reviews In Biochemistry and Molecular Biology 48(2):123-152. https://doi.org/10.3109/10409238.2012.758083

Torabi B, Soltani E, Archontoulis SV, Rabii A (2016). Temperature and water potential effects on Carthamus tinctorius L. seed germination: measurements and modeling using hydrothermal and multiplicative approaches. Brazilian Journal of Botany 39(2):427-436. https://doi.org/10.1007/s40415-015-0243-X

Tripathi R, Agrawal S (2013). Evaluation of changes in lipid peroxidation, ROS production, surface structures, secondary metabolites and yield of linseed (Linum usitatissimum L.) under individual and combined stress of ultraviolet-B and ozone using open top chambers. Indian Journal of Biochemistry and Biophysics 50(4):318-325.

Tsai CJ, Harding SA, Tschaplinski TJ, Lindroth RL, Yuan Y (2006). Genome-wide analysis of the structural genes regulating defense phenylpropanoid metabolism in Populus. New Phytologist 172(1):47-62. https://doi.org/10.1111/j.1469-8137.2006.01798.x

Vanholme B, Cesarino I, Goeminne G, Kim H, Marroni F, Van Acker R, ... Pinosio S (2013). Breeding with rare defective alleles (BRDA): a natural Populus nigra HCT mutant with modified lignin as a case study. New Phytologist 198(3):765-776. https://doi.org/10.1111/nph.12179

Vanholme R, De Meester B, Ralph J, Boerjan W (2019). Lignin biosynthesis and its integration into metabolism. Current Opinion in Biotechnology 56:230-239. https://doi.org/10.1016/j.copbio.2019.02.018

Varbanova M, Porter K, Lu F, Ralph J, Hammerschmidt R, Jones AD, Day B (2011). Molecular and biochemical basis for stress-induced accumulation of free and bound p-coumaraldehyde in cucumber. Plant Physiology 157(3):10561066. https://doi.org/10.1104/pp.111.184358

Velasco R, Zharkikh A, Affourtit J, Dhingra A, Cestaro A, Kalyanaraman A, ... Pruss D (2010). The genome of the domesticated apple (Malus $\times$ domestica Borkh.). Nature Genetics 42(10):833. https://doi.org/10.1038/ng.654

Verde I, Abbott AG, Scalabrin S, Jung S, Shu S, Marroni F, ... Cattonaro F (2013). The high-quality draft genome of peach (Prunus persica) identifies unique patterns of genetic diversity, domestication and genome evolution. Nature Genetics 45(5):487. https://doi.org/10.1038/ng.2586

Wagner A, Ralph J, Akiyama T, Flint H, Phillips L, Torr K, Nanayakkara B, Te Kiri L (2007). Exploring lignification in conifers by silencing hydroxycinnamoyl-CoA: shikimate hydroxycinnamoyltransferase in Pinus radiata. Proceedings of the National Academy of Sciences 104(28):11856-11861. https://doi.org/10.1073/pnas.0701428104

Wang GF, He Y, Strauch R, Olukolu BA, Nielsen D, Li X, Balint-Kurti PJ (2015). Maize homologs of hydroxycinnamoyltransferase, a key enzyme in lignin biosynthesis, bind the nucleotide binding leucine-rich repeat Rp1 proteins to modulate the defense response. Plant Physiology 169(3):2230-2243.

Weng JK, Chapple C (2010). The origin and evolution of lignin biosynthesis. New Phytologist 187(2):273-285. https://doi.org/10.1111/j.1469-8137.2010.03327.x

Wu J, Wang Z, Shi Z, Zhang S, Ming R, Zhu S, Khan MA, Tao S, Korban SS, Wang H (2013). The genome of the pear (Pyrus bretschneideri Rehd.). Genome Research 23(2):396-408. https://doi.org/10.1101/gr.144311.112

Xu Z, Zhang D, Hu J, Zhou X, Ye X, Reichel KL, ... Gao P (2009). Comparative genome analysis of lignin biosynthesis gene families across the plant kingdom. BMC Bioinformatics 10(11):1-15. https://doi.org/10.1186/1471-210510-S11-S3

Ye SY, Gao WY (2008). Hydroxysafflor yellow A protects neuron against hypoxia injury and suppresses inflammatory responses following focal ischemia reperfusion in rats. Archives of Pharmacal Research 31(8):1010-1015. https://doi.org/10.1007/s12272-001-1261-y 
Zhang Y, Guo J, Dong H, Zhao X, Zhou L, Li X, Liu J, Niu Y (2011). Hydroxysafflor yellow A protects against chronic carbon tetrachloride-induced liver fibrosis. European Journal of Pharmacology 660(2-3):438-444. https://doi.org/10.1016/j.ejphar.2011.04.015

OPEN ACCESS

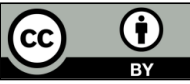

The journal offers free, immediate, and unrestricted access to peer-reviewed research and scholarly work. Users are allowed to read, download, copy, distribute, print, search, or link to the full texts of the articles, or use them for any other lawful purpose, without asking prior permission from the publisher or the author.

License - Articles published in Notulae Botanicae Horti Agrobotanici Cluj-Napoca are Open-Access, distributed under the terms and conditions of the Creative Commons Attribution (CC BY 4.0) License.

(C) Articles by the authors; UASVM, Cluj-Napoca, Romania. The journal allows the author(s) to hold the copyright/to retain publishing rights without restriction. 


\section{Genome-wide investigation of Hydroxycinnamoyl CoA: Shikimate Hydroxycinnamoyl Transferase (HCT) gene family in Carthamus tinctorius $L$.}

\section{Supplementary files}

Table S1. Primers set used in qRT-PCR analyses

\begin{tabular}{|c|c|c|}
\hline Primer & Strand & Primer sequence $\left(5^{\prime}-3^{\prime}\right)$ \\
\hline \multirow{2}{*}{ CtHCT001 } & Forward & CGGAACATCTTTCTGGCATT \\
\hline & Reverse & ATTGTCATTTTCCCGTCGTC \\
\hline \multirow{2}{*}{ CtHCT005 } & Forward & AGCTCACCATTTCCCCAATA \\
\hline & Reverse & AAAGGCTGAAGATGGGGTTT \\
\hline \multirow{2}{*}{ CtHCT009 } & Forward & TTCGAGGTCCATCCTCAAAC \\
\hline & Reverse & ACAGTCCTTCCTCGTTGGTG \\
\hline \multirow{2}{*}{ CtHCT017 } & Forward & CAGCCATCCAAGGATACGAT \\
\hline & Reverse & CCTCGTCGTCTTCAAGATCC \\
\hline \multirow{2}{*}{ CtHCT019 } & Forward & TGAGCTTAACGACGGGATCT \\
\hline & Reverse & СТТТССССАAАССАAАСТСА \\
\hline \multirow{2}{*}{ CtHCT027 } & Forward & CTTATTCCGGCGGTTGATTA \\
\hline & Reverse & GGCTGCAGTTCCAAGAAATC \\
\hline \multirow{2}{*}{ CtHCT028 } & Forward & АСССТССССААТСТСАААСТ \\
\hline & Reverse & TCGGAGACGGTAATTTGGTC \\
\hline \multirow{2}{*}{ CtHCT030 } & Forward & TCCCCGATCTCGATACTTTG \\
\hline & Reverse & TATTTTGCTCGCGATGTGTC \\
\hline \multirow{2}{*}{ СtHCT031 } & Forward & CGATCCACCAGGTTTTCTTC \\
\hline & Reverse & TGTTGGGTCCGTAACCATTT \\
\hline \multirow{2}{*}{ CtHCT033 } & Forward & CCAAAGAGCAAGGACGTTGT \\
\hline & Reverse & CCGGTAAAAGGCAAACTTCA \\
\hline \multirow{2}{*}{ СtHCT036 } & Forward & GTTTCTTGTGGAAGGGGTCA \\
\hline & Reverse & CCGAAGTCCATTCCGTAAAA \\
\hline \multirow{2}{*}{ CtHCT039 } & Forward & ACTTCTCAGGGATGCCACAA \\
\hline & Reverse & ACTCTCGGCACCTTTCAAGA \\
\hline \multirow{2}{*}{ CtHCT040 } & Forward & CAGGCTTAGACCCGACCATA \\
\hline & Reverse & CCTTCCGAATACGTCTCTGC \\
\hline \multirow{2}{*}{ СtHCT048 } & Forward & AAATGTGGAGGCATGGTGAT \\
\hline & Reverse & TCACCGACGTTTCTTTCTCC \\
\hline \multirow{2}{*}{ CtHCT055 } & Forward & CAAGACTCCCTTGACCTTCG \\
\hline & Reverse & GACCTATGGGCCATGTCATC \\
\hline \multirow{2}{*}{ CtHCT059 } & Forward & ACCGTTGTTATCCGTTCAGG \\
\hline & Reverse & CTCCCGTACATGTCGAACCT \\
\hline \multirow{2}{*}{ CtHCT065 } & Forward & CTTCCСТTCCCATCTTGACA \\
\hline & Reverse & GTTTCAACCTTGGCGTGTTT \\
\hline \multirow{2}{*}{ CtHCT069 } & Forward & AGTTTCGAGCGGTCTTGTGT \\
\hline & Reverse & CCATGGTTCCTTTGGAGCTA \\
\hline \multirow{2}{*}{ СtHCT077 } & Forward & GGATCTCACCACCACCAAAC \\
\hline & Reverse & GTCTGGCTAACCAGCAGAGG \\
\hline \multirow{2}{*}{ СtHCT081 } & Forward & TGACGAATGTCCGAGTGAAC \\
\hline & Reverse & CСССАТСТТАСАССАACTCG \\
\hline
\end{tabular}


Fan S et al. (2021). Not Bot Horti Agrobo 49(3):12489

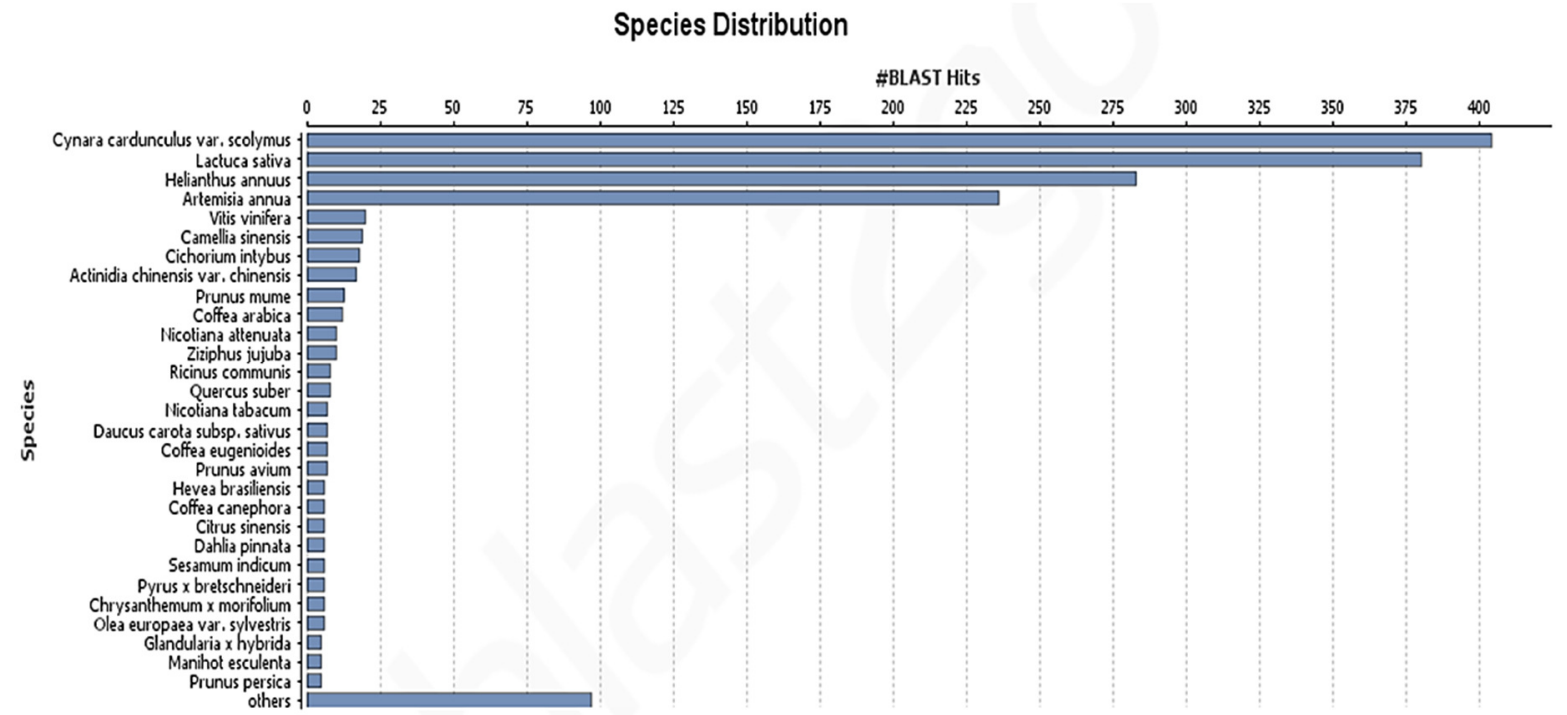

Figure S1. Distribution of HCT gene family shared by different plant species

(A)

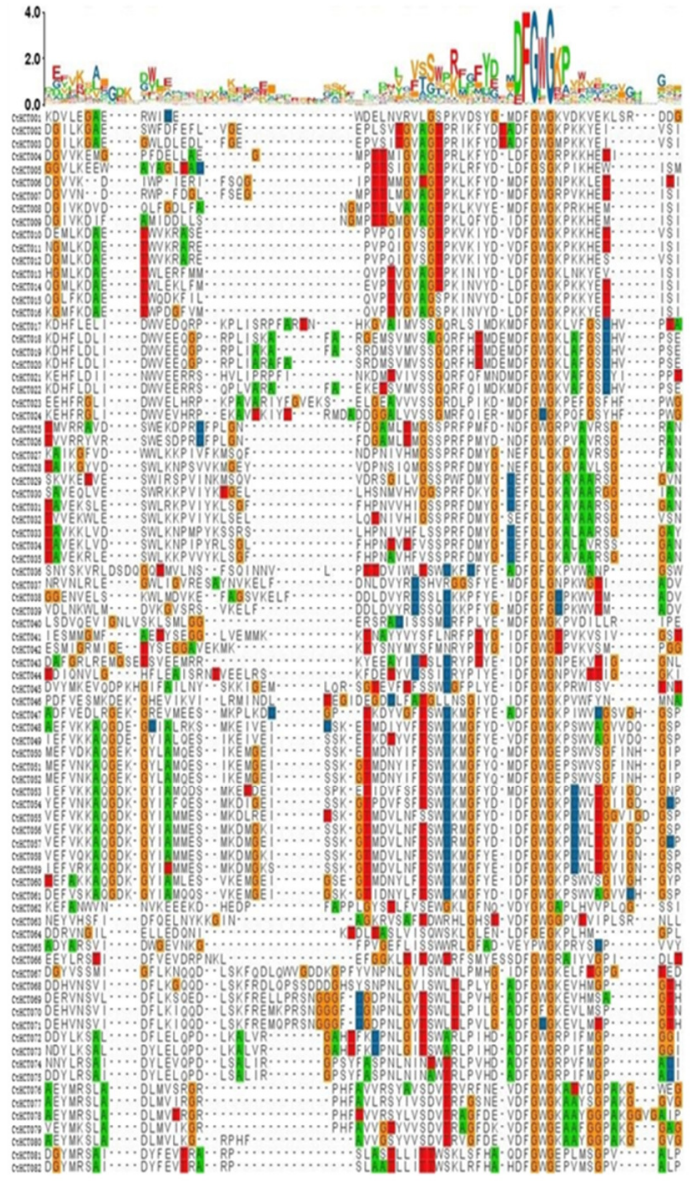

(B)

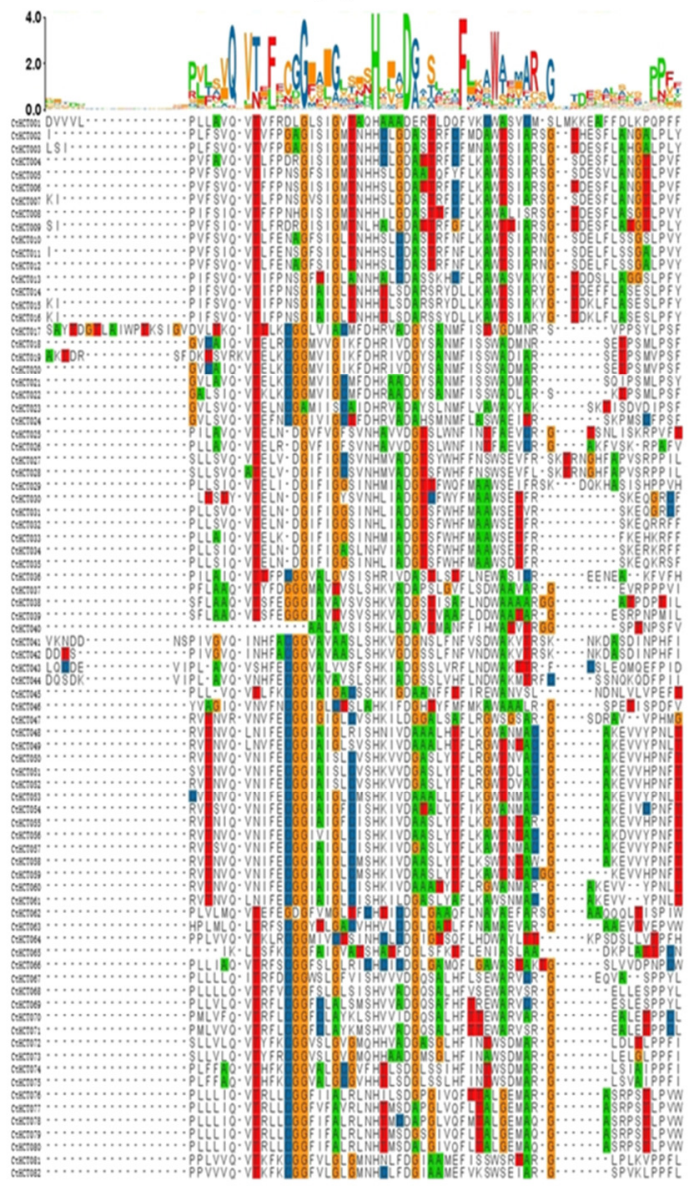

Figure S2. Multiple sequence alignment of the $82 \mathrm{CtHCT}$ proteins

(A-B) The Logos of the two widespread conserved HCT domains of Carthamus tinctorius consisting HXXXD and DFGWG amino acids and the pairwise alignment of Carthamus tinctorius HCT proteins. The different colored boxes represent $60 \%$ similarity of the conserved amino acid residues 

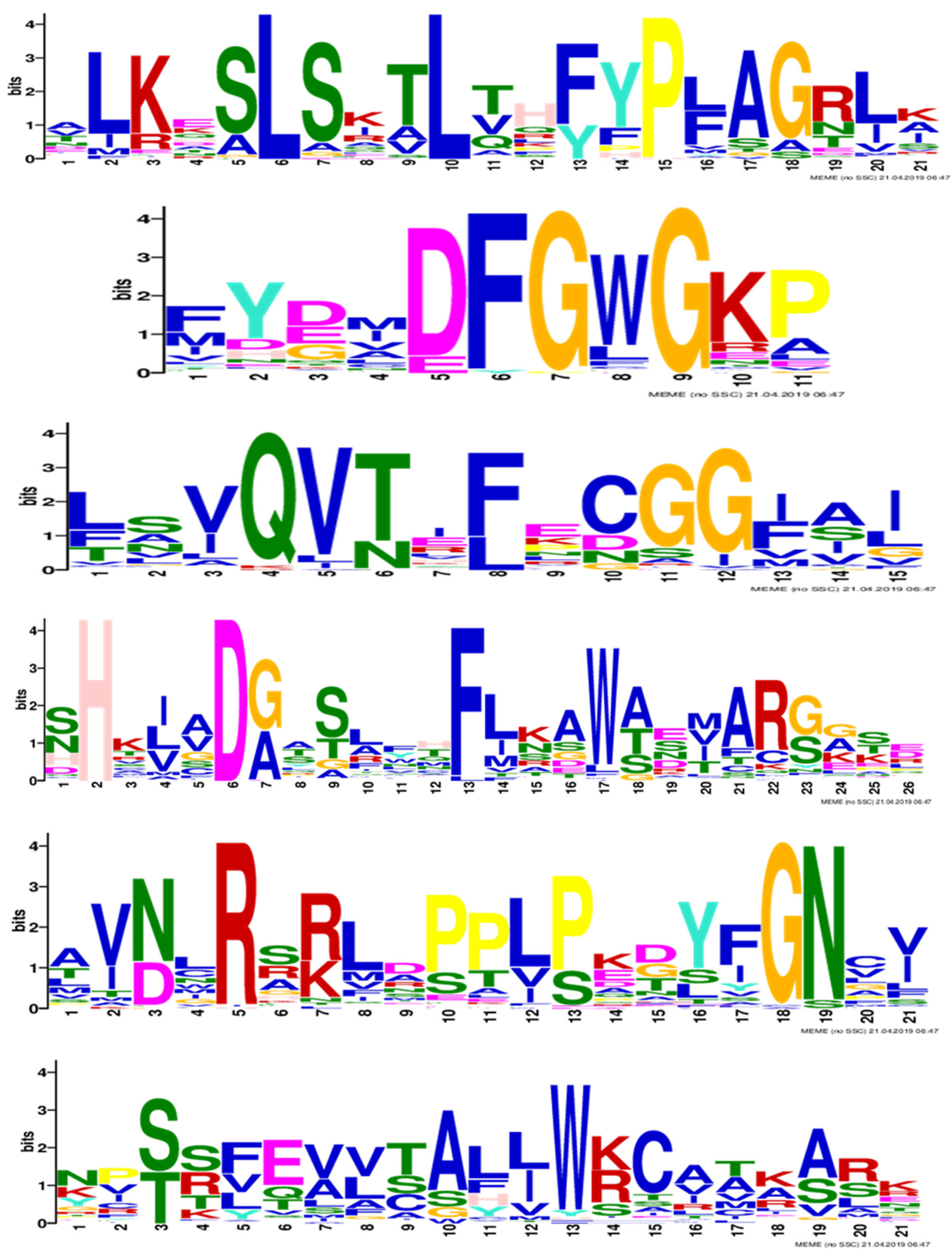

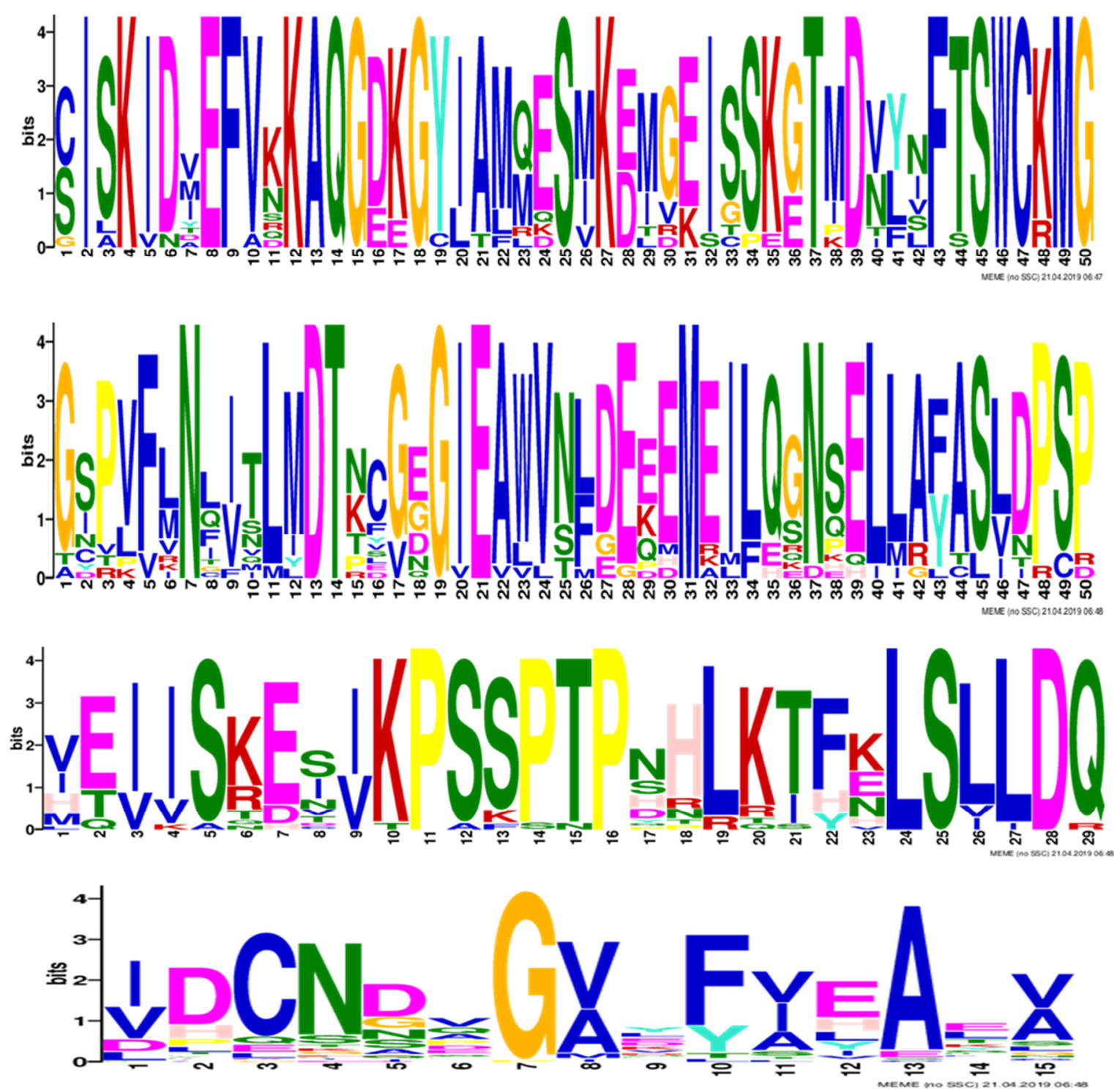

Figure S3. Logos of the 10 conserved protein motifs within CtHCT proteins obtained from the MEME online web-server 
(A)

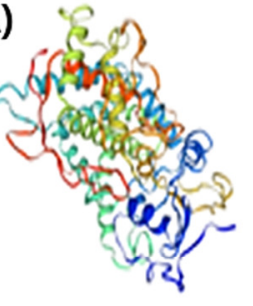

(B)

$\mathrm{CAH}$

(F)

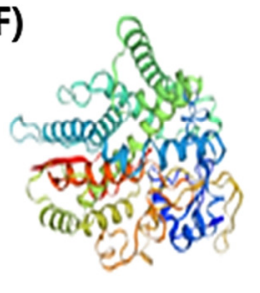

CYP98A3
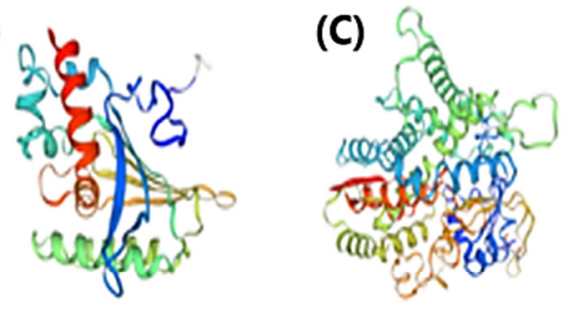

CHIL

(G)

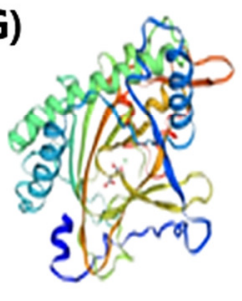

(D)

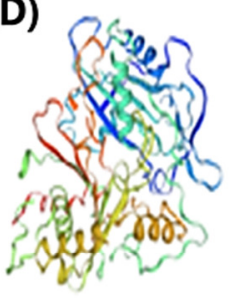

HCT

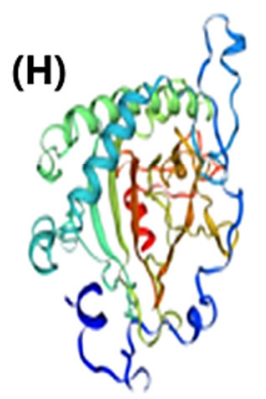

FLS1

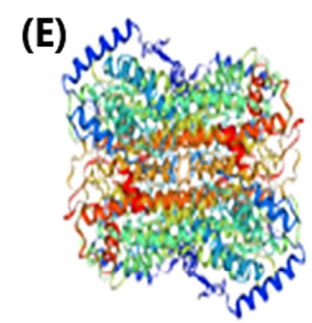

PAL1

Figure S4. The three-dimensional structures of the ten putative interactor-proteins with a member of CtHCT putative protein 

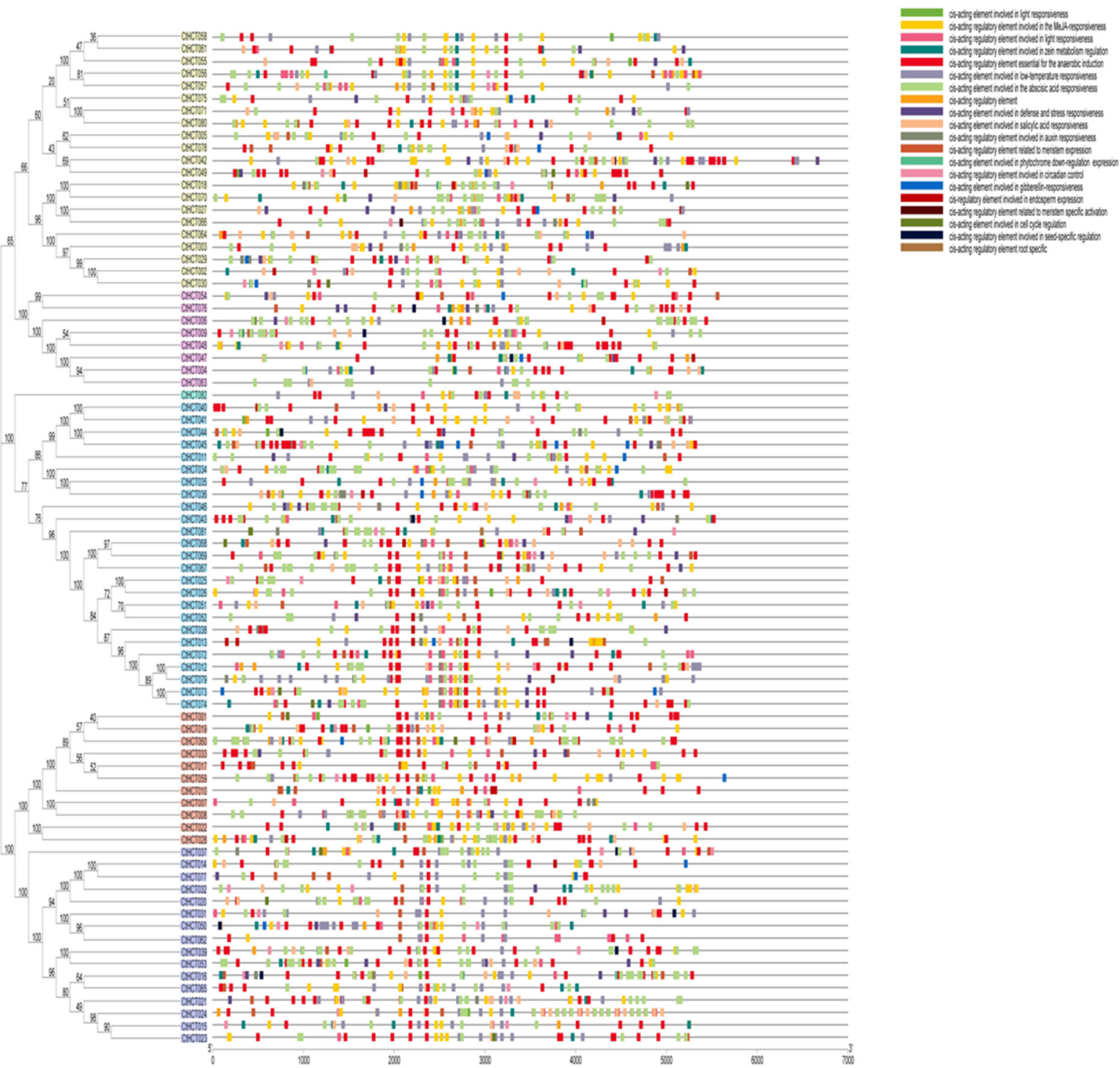

Figure S5. The graphical representation of the twenty conserved cis-regulatory elements identified in the 5' untranslated region ( 5 ' UTR) of the promoter sequence of CtHCT genes

Each colour represents a different type of cis-acting element within the promotor site of $C t H C T$ genes. 


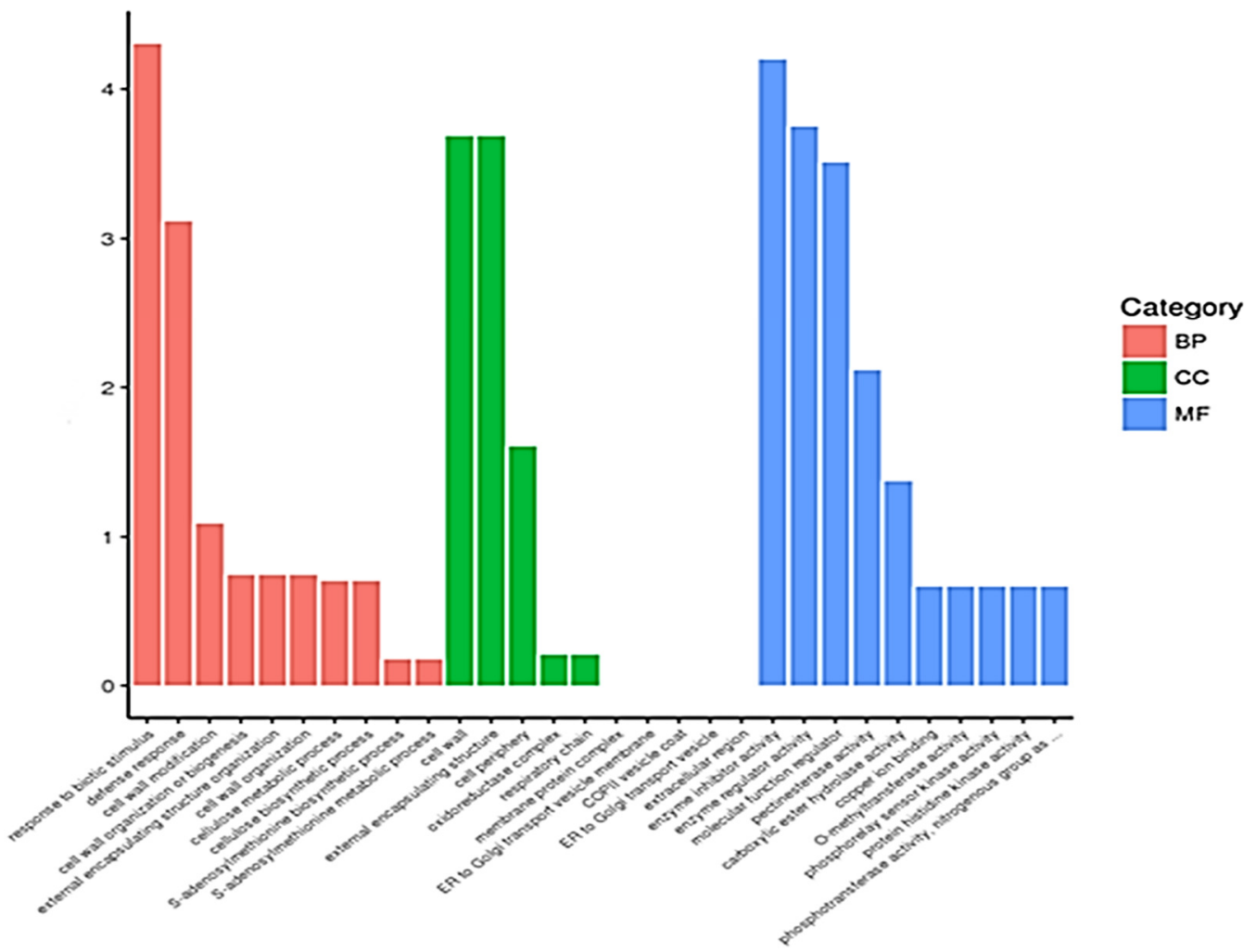

Figure S6. The Go term classification of Carthamus tinctorius HCTs

The annotation was investigated across three main categories: (a) biological process, (b) cellular component and (c) molecular function. The coloured boxes indicate pathways enrichment (in blue) and cellular component (yellow) with per cent score. 

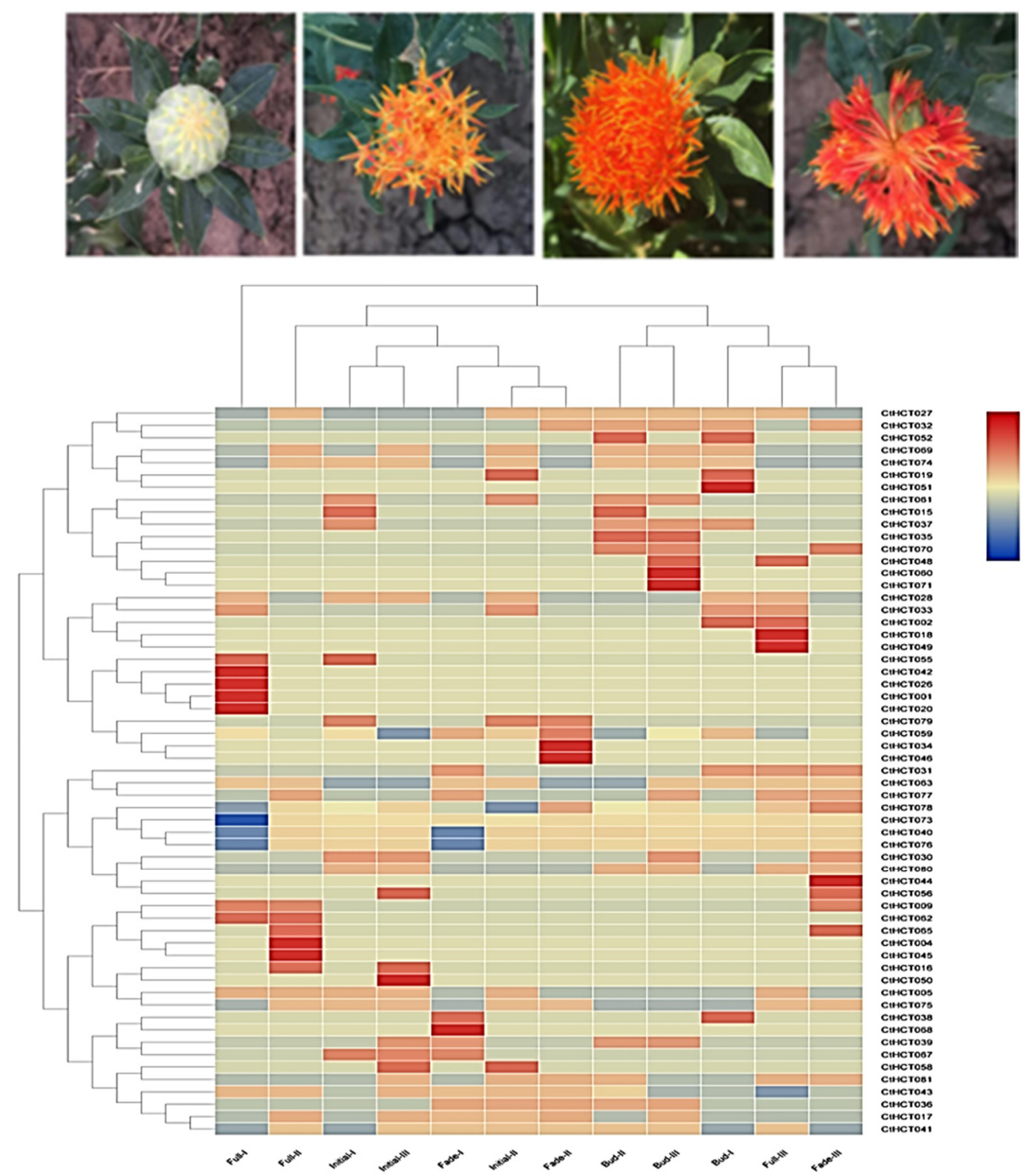

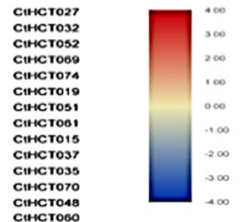

Figure S7. The four different flower developmental stages of Carthamus tinctorius and the expression profile of CtHCT genes

The heatmap was generated from the FPKM data obtained from RNA-seq data. 